\title{
QRAP: A numerical code for projected (Q)uasiparticle (RA)ndom (P)hase approximation ${ }^{\hbar}$
}

\author{
A.R. Samana ${ }^{\text {a,b }}$, F. Krmpotićc,d, C.A. Bertulani ${ }^{\text {a,* }}$ \\ a Department of Physics, Texas A\&M University Commerce, P.O. 3011, Commerce, TX 75429, USA \\ b Departamento de Ciências Exactas e Tecnológicas, Universidade Estadual de Santa Cruz, CEP 45662-000 Ilhes, Bahia-BA, Brazil \\ c Instituto de Física La Plata, CONICET, 1900 La Plata, Argentina \\ d Facultad de Ciencias Astronómicas y Geofísicas, Universidad Nacional de La Plata, 1900 La Plata, Argentina
}

\section{A R T I C L E I N F O}

\section{Article history:}

Received 23 June 2009

Received in revised form 22 January 2010

Accepted 2 February 2010

Available online 4 February 2010

\section{Keywords:}

QRPA

Projected QRPA

Semileptonic processes

\begin{abstract}
A B S T R A C T
A computer code for quasiparticle random phase approximation - QRPA and projected quasiparticle random phase approximation - PQRPA models of nuclear structure is explained in details. The residual interaction is approximated by a simple $\delta$-force. An important application of the code consists in evaluating nuclear matrix elements involved in neutrino-nucleus reactions. As an example, cross sections for ${ }^{56} \mathrm{Fe}$ and ${ }^{12} \mathrm{C}$ are calculated and the code output is explained. The application to other nuclei and the description of other nuclear and weak decay processes are also discussed.
\end{abstract}

\section{Program summary}

Title of program: QRAP (Quasiparticle RAndom Phase approximation)

Computers: The code has been created on a PC, but also runs on UNIX or LINUX machines Operating systems: WINDOWS or UNIX

Program language used: Fortran-77

Memory required to execute with typical data: 16 Mbytes of RAM memory and $2 \mathrm{MB}$ of hard disk space No. of lines in distributed program, including test data, etc.: $\sim 8000$

No. of bytes in distributed program, including test data, etc.: $\sim 256 \mathrm{kB}$

Distribution format: tar.gz

Nature of physical problem: The program calculates neutrino- and antineutrino-nucleus cross sections as a function of the incident neutrino energy, and muon capture rates, using the QRPA or PQRPA as nuclear structure models.

Method of solution: The QRPA, or PQRPA, equations are solved in a self-consistent way for even-even nuclei. The nuclear matrix elements for the neutrino-nucleus interaction are treated as the beta inverse reaction of odd-odd nuclei as function of the transfer momentum.

Typical running time: $\approx 5 \mathrm{~min}$ on a $3 \mathrm{GHz}$ processor for Data set 1 .

(C) 2010 Elsevier B.V. All rights reserved.

\section{Introduction}

The new age of the physics beyond the standard model of electroweak interaction has as one of the most promising pathways the search of neutrino oscillations. Several experimental efforts are oriented to find the neutrino masses and the related oscillations involving atmospheric, solar, reactor and accelerator neu-

\footnotetext{
is This paper and its associated computer program are available via the Computer Physics Communications homepage on ScienceDirect (http://www.sciencedirect. com/science/journal/00104655).

* Corresponding author.

E-mail addresses: arturo_samana@tamu-commerce.edu (A.R. Samana), krmpotic@fisica.unlp.edu.ar (F. Krmpotić),carlos_bertulani@tamu-commerce.edu (C.A. Bertulani).
}

trinos [1-5]. Since neutrinos interact so weakly with matter, they bring information on the dynamics of supernova collapse and posterior explosion as well as on the synthesis of heavy nuclei [6,7].

The detection signal of neutrinos is measured trough the weak interaction of incoming neutrinos with the nuclei present in, e.g., a liquid scintillator detector, as well as with the surrounding blockhouse detector shield. The flux-averaged $v$-nucleus cross sections are the measured observables. Recently, Ref. [8] has studied the effect of neutrino oscillations on the expected supernova neutrino signal with the LVD detector, through their interactions with protons and carbon nuclei in a liquid scintillator and with iron nuclei in the support structure.

Charged and neutral $v_{e}$-nucleus cross sections on ${ }^{12} \mathrm{C}$ (liquid scintillator) as well as on ${ }^{56} \mathrm{Fe}$ (detector surrounding shield) were 
measured by the KARMEN Collaboration $[9,10]$. Other experiments such as LAMPF [11,12] and LSND [13,14] have also used ${ }^{12} \mathrm{C}$ to search for neutrino oscillations and to measure neutrino-nucleus cross sections. Furthermore, future experiments will use ${ }^{12} \mathrm{C}$ as liquid scintillator, such as in the spallation neutron source (SNS) at Oak Ridge National Laboratory (ORNL) [15], or in the LVD (Large Volume Detector) experiment [8].

On the other hand, the cross sections $v_{e}\left(\bar{v}_{e}\right)-{ }^{56} \mathrm{Fe}$ are important to test the ability of nuclear models in explaining reactions on nuclei with masses around iron, which play an important role in supernova collapse [16]. The iron is used as material detector in experiments on neutrino oscillations such as MINOS [17], whereas future experiments, such as SNS at ORNL [15] plan to use the same material.

There have been great efforts on nuclear structure models to describe consistently semileptonic weak processes with ${ }^{12} \mathrm{C}$ such as RPA-like models. A brief summary on the different models employed for ${ }^{12} \mathrm{C}$ is sketched in Ref. [18].

The puzzle with the Random Phase Approximation (RPA) and the quasiparticle RPA (QRPA), when applied to the weak observables in the triad $\left\{{ }^{12} \mathrm{~B},{ }^{12} \mathrm{C},{ }^{12} \mathrm{~N}\right\}$, is well known. That is, to get agreement with data for the ground state triplet $T=1\left(\beta^{ \pm}\right.$-decays, $\mu$-capture, and the exclusive ${ }^{12} \mathrm{C}\left(v_{e}, e^{-}\right){ }^{12} \mathrm{~N}$ reaction) the continuum RPA (CRPA) calculations of Kolbe, Langanke, and Krewald [19] needed to be rescaled by a reduction factor $\cong 4$. The reason for such a large discrepancy is very simple: within the RPA the transitions ${ }^{12} \mathrm{C} \rightarrow{ }^{12} \mathrm{~N}\left(1_{1}^{+}\right)$and ${ }^{12} \mathrm{C} \rightarrow{ }^{12} \mathrm{~B}\left(1_{1}^{+}\right)$are engendered mostly by the particle-hole excitation $p_{3 / 2} \rightarrow p_{1 / 2}$, what is physically incorrect. In fact, since late 1980's we know from several hadronic charge-exchange reaction measurements, and the consecutive Shell Model (SM) calculations, that the excitations $p_{3 / 2} \rightarrow p_{3 / 2}, p_{1 / 2} \rightarrow$ $p_{1 / 2}$, and $p_{1 / 2} \rightarrow p_{3 / 2}$ participate quite significantly in these processes (see, for instance, [20, Table I]). It is the involvement of these configurations that brings about the necessary quenching of the Gamow-Teller (GT) resonances and $\beta$-decay rates. To make them come into play it is mandatory to open the $p_{3 / 2}$ shell by means of pairing correlations, which is done within both the SM and the QRPA. But, a new problem emerges in the application of the QRPA to ${ }^{12} \mathrm{C}$, as first observed by Volpe et al. [21] who noted that within this approach the lowest state in ${ }^{12} \mathrm{~N}$ irremediable turned out not to be the most collective one. As a consequence the QRPA also fails in accounting for the exclusive processes to the isospin triplet $T=1$. Soon after it was shown [22-24] that the origin of this difficulty arises from the degeneracy among the $p_{1 / 2}$ and $p_{3 / 2}$ quasiparticle energies (both for protons and neutrons), which is inherent to the non-conservation of particle number. Therefore, for a physically sound description of the weak processes among the $A=12$ iso-triplet it is imperative to use the SM or the number projected QRPA (PQRPA).

The QRAP code is based on Refs. [22-24], where a new formalism for neutrino-nucleus scattering has been developed, and the PQRPA is used as the nuclear model framework. The residual interaction was done with the simple $\delta$-force, which has been used extensively in the literature to describe the single and double beta decays [25-30].

Before proceeding we address briefly on the genesis of the QRPA and PQRPA in a manner appropriate in the present context. Although this is not a topic of central interest for the applicationoriented computer code, it belongs to the physics background. The neutron-proton QRPA was developed in 1967 by Hableib and Sorensen [31] in order to account for the hindrance of the allowed $\beta$-transitions. Almost 20 years later, when Vogel and Zirnbauer [32] and Cha [33] discovered the importance of the particleparticle force in the $S=1, T=0$ channel, the QRPA became to be the most frequently used nuclear structure method for evaluating double beta $(\beta \beta)$ rates. It was quickly realized, however, that a small change in the particle-particle interaction strength caused a large change in the lifetimes and eventually the breakdown (called a "collapse") of the entire method. Later on several modifications of the QRPA were proposed to make it more reliable. One of these was the charge-exchange PQRPA, which has been formulated to evade the disadvantages inherent in the non-conservation of particle number, and was derived from the time-dependent variational principle [29]. But, the PQRPA did not yield substantially different result from the plain QRPA, and was unable to avoid the collapse in the study the two-neutrino $\beta \beta$-decay in ${ }^{76} \mathrm{Ge}$. As a matter of fact, the problem of the QRPA collapse has not yet been settled down, in spite of enormous effort invested for this purpose by many nuclear physicists (compare, for instance, Fig. 1 from Ref. [29] with Fig. 5 from a recent work of Yousef et al. [34]).

However, the PQRPA turned out to be quite important for the description of relatively light nuclei such as ${ }^{12} \mathrm{C}$. For example, the employment of PQRPA for the inclusive ${ }^{12} \mathrm{C}\left(v_{e}, e^{-}\right)^{12} \mathrm{~N}$ cross section, instead of the continuum RPA (CRPA) used by the LSND Collaboration in the analysis of $v_{\mu} \rightarrow v_{e}$ oscillations of the 1993-1995 data sample, leads to an increased oscillation probability [24].

The PQRPA was recently also used to calculate the ${ }^{56} \mathrm{Fe}\left(v_{e}, e^{-}\right)$${ }^{56} \mathrm{Co}$ cross section [35]. A comparison between the QRPA and PQRPA for the same interaction and employing the same model space shows that the projection procedure could be important for medium mass nuclei. Moreover, several approximations such as: i) Hybrid Model (HM) [36], ii) QRPA with Skyrme interaction [37], iii) relativistic QRPA (RQRPA) [38], and iv) QRPA and PQRPA with the $\delta$-force [35] yield different results for the neutrino cross section as a function of the neutrino energy. It is a hard task to find the origin for the differences, mainly because these models are not using the same interaction and/or the same single-particle configuration space, carrying different types of correlations in each case.

The cross sections for charged- and neutral-current neutrinoinduced reactions on the iron isotopes ${ }^{52-60} \mathrm{Fe}$ were also evaluated within the HM for various supernova neutrino spectra [39]. Here, large-scale SM calculations were used for the GT-like contributions, while transitions for other multipoles are based on the RPA. More precisely, the authors scale the SM cross sections using the ratios obtained from the RPA calculations with and without this dependence of the multipole operator. The reason for such a procedure is twofold: i) the limitation of the SM to account for momentumtransfer dependence of the GT operator, and ii) the lack of pairing correlations in the RPA. It should be also mentioned that SM calculations of inelastic neutral-current neutrino-nucleus cross sections in medium-mass nuclei, present in supernova environment, have been constrained by the highly precise data on the magnetic dipole strength distributions for the nuclei ${ }^{50} \mathrm{Ti},{ }^{52} \mathrm{Cr}$, and ${ }^{54} \mathrm{Fe}$, which are dominated by spin-isospin flipping (GT-like) contributions [40]. In spite of the agreement between data and calculations it was necessary to consider also here the effects of finite momentum transfer what was done via the RPA. Briefly, the HM is neither fish nor fowl, and a comparison of the results from Refs. $[39,40]$ with selfconsistent calculations, such as the QRPA, PQRPA and RQRPA, could be enlightening.

This brief introduction shows: 1) the importance of neutrinonucleus cross sections for astrophysical purposes, and 2) that these cross sections are strongly correlated with the nuclear structure model employed. The QRAP code, with a simple residual interaction, is able to access the sources of these problems and it can calculate several weak interaction processes mentioned above. Needless to stress that this code can be easily adapted for the evaluation of $\beta \beta$-decays.

The write-up is organized as follows. In Section 2 we make a short survey of the theoretical description of weak interaction processes, with emphasis on the formulation implemented in this numerical code. In Section 3 we describe the QRPA, and PQRPA for- 
malisms, making explicit the differences among them. In Section 4 we show how the code is organized, how to make an input and how to understand the output. Section 5 explains the role of each subroutine of the code. Finally, Section 6 proposes a few cases to practice with the code.

\section{Weak interacting processes}

In this section we give a brief summary of the main formulae developed in Refs. [18,23] for:

- neutrino scattering (NS)

$$
v_{\ell}+(Z, A) \rightarrow(Z+1, N-1)+\ell^{-},
$$

- antineutrino scattering (AS)

$$
\bar{v}_{\ell}+(Z, A) \rightarrow(Z-1, N+1)+\ell^{+},
$$

- muon capture (MC) rate

$$
\mu^{-}+(Z, A) \rightarrow(Z-1, N-1)+v_{\mu}
$$

where $\ell=e, \mu$. The comparison with other formalisms [41-43] can be found is in just mention works.

The weak Hamiltonian is expressed in the form

$H_{W}(\mathbf{r})=\frac{G}{\sqrt{2}} J_{\alpha} l_{\alpha} e^{-i \mathbf{r} \cdot \mathbf{k}}$,

where $G=(3.04545 \pm 0.00006) \times 10^{-12}$ is the Fermi coupling constant (in natural units),

$$
\begin{aligned}
J_{\alpha} & \equiv\left(\mathbf{J}, i J_{\emptyset}\right) \\
& =i \gamma_{4}\left[g_{V} \gamma_{\alpha}-\frac{g_{M}}{2 \mathrm{M}} \sigma_{\alpha \beta} k_{\beta}+g_{A} \gamma_{\alpha} \gamma_{5}+i \frac{g_{P}}{\mathrm{~m}_{\ell}} k_{\alpha} \gamma_{5}\right],
\end{aligned}
$$

is the hadronic current operator, ${ }^{1}$ and

$$
l_{\alpha}\left(\mathbf{q}, E_{v}\right) \equiv\left(\mathbf{l}, i l_{\emptyset}\right)=-i \bar{u}_{s_{\ell}}\left(\mathbf{p}, E_{\ell}\right) \gamma_{\alpha}\left(1+\gamma_{5}\right) u_{s_{v}},
$$

is the plane wave approximation for the matrix element of the leptonic current in the case of neutrino reactions, with $p_{\ell} \equiv\left\{\mathbf{p}, i E_{\ell}\right\}$ and $q_{v} \equiv\left\{\mathbf{q}, i E_{v}\right\}$ being, respectively, the lepton and the neutrino momenta.

For the sake of convenience we will use spherical coordinates ( $m=-1,0,+1)$ for the three-vectors, and the Walecka's notation [42], with the Euclidean metric, for four-vectors, i.e., $x=\left\{\mathbf{x}, x_{4}=\right.$ $\left.i x_{\emptyset}\right\}$. The only difference is that we substitute Walecka's indices $(0,3)$ by our indices $(\emptyset, 0)$, i.e. we use the index $\emptyset$ for the temporal component and the index 0 for the third spherical component.

The quantity

$k=P_{i}-P_{f} \equiv\left\{\mathbf{k}, i k_{\emptyset}\right\}$,

is the momentum transfer, where $P_{i}$ and $P_{f}$ are momenta of the initial and final nucleus, $M$ is the nucleon mass, $m_{\ell}$ is the mass of the charged lepton, and $g_{V}, g_{A}, g_{M}$ and $g_{P}$ are, respectively, the vector, axial-vector, weak-magnetism and pseudoscalar effective dimensionless coupling constants. Their numerical values are:

$$
\begin{aligned}
& g_{V}=1 ; \quad g_{A}=1.26 ; \\
& g_{M}=\kappa_{p}-\kappa_{n}=3.70 ; \quad g_{P}=g_{A} \frac{2 \mathrm{Mm}_{\ell}}{k^{2}+\mathrm{m}_{\pi}^{2}} .
\end{aligned}
$$

\footnotetext{
1 To avoid confusion, we will be using roman fonts $(\mathrm{M}, \mathrm{m})$ for masses and math italic fonts $(M, m)$ for azimuthal quantum numbers.
}

In the numerical calculations we use an effective axial-vector coupling $g_{A}=1$ [44].

The finite nuclear size (FNS) effect is incorporated via the dipole form factor with a cutoff $\Lambda=850 \mathrm{MeV}$, i.e.,

$$
g \rightarrow g\left(\frac{\Lambda^{2}}{\Lambda^{2}+k^{2}}\right)^{2} \text {. }
$$

To use (2.1) with the non-relativistic nuclear wave functions, the Foldy-Wouthuysen transformation has to be performed on the hadronic current (2.2). When the velocity-dependent terms are included this yields [45]:

$$
\begin{aligned}
& J \emptyset=g_{\mathrm{V}}+\left(\bar{g}_{\mathrm{A}}+\bar{g}_{\mathrm{P} 1}\right) \boldsymbol{\sigma} \cdot \hat{\mathbf{k}}-g_{\mathrm{A}} \boldsymbol{\sigma} \cdot \mathbf{v}, \\
& \mathbf{J}=-g_{\mathrm{A}} \boldsymbol{\sigma}-i \bar{g}_{\mathrm{W}} \boldsymbol{\sigma} \times \hat{\mathbf{k}}-\bar{g}_{\mathrm{V}} \hat{\mathbf{k}}+\bar{g}_{\mathrm{P} 2}(\boldsymbol{\sigma} \cdot \hat{\mathbf{k}}) \hat{\mathbf{k}}+g_{\mathrm{V}} \mathbf{v},
\end{aligned}
$$

where $\hat{\mathbf{k}}=\mathbf{k} / \kappa, \kappa \equiv|\mathbf{k}|$, and $\mathbf{v} \equiv-i \nabla / \mathrm{M}$ is the velocity operator, acting on the nuclear wave functions. The following short notation

$$
\begin{aligned}
& \bar{g}_{\mathrm{V}}=g_{\mathrm{V}} \frac{\kappa}{2 \mathrm{M}} ; \quad \bar{g}_{\mathrm{A}}=g_{\mathrm{A}} \frac{\kappa}{2 \mathrm{M}} ; \quad \bar{g}_{\mathrm{W}}=\left(g_{\mathrm{V}}+g_{\mathrm{M}}\right) \frac{\kappa}{2 \mathrm{M}}, \\
& \bar{g}_{\mathrm{P} 1}=g_{\mathrm{P}} \frac{\kappa}{2 \mathrm{M}} \frac{q_{\emptyset}}{\mathrm{m}_{\ell}} ; \quad \bar{g}_{\mathrm{P} 2}=g_{\mathrm{P}} \frac{\kappa}{2 \mathrm{M}} \frac{\kappa}{\mathrm{m}_{\ell}},
\end{aligned}
$$

has also been introduced.

In performing the multipole expansion of the nuclear operators

$$
O_{\alpha} \equiv\left(\mathbf{O}, O_{\emptyset}\right)=J_{\alpha} e^{-i \mathbf{k} \cdot \mathbf{r}},
$$

it is convenient:

1) to take the momentum $\mathbf{k}$ to be along the $z$ axis, i.e.,

$$
\begin{aligned}
e^{-i \mathbf{k} \cdot \mathbf{r}} & =\sum_{\mathrm{L}} i^{-\mathrm{L}} \sqrt{4 \pi(2 \mathrm{~L}+1)} j_{\mathrm{L}}(\rho) Y_{\mathrm{L} 0}(\hat{\mathbf{r}}), \\
& =\sum_{\mathrm{J}} i^{-\mathrm{J}} \sqrt{4 \pi(2 \mathrm{~J}+1)} j_{\mathrm{J}}(\rho) Y_{\mathrm{J} 0}(\hat{\mathbf{r}}),
\end{aligned}
$$

where $\rho=\kappa r$, and

2) to introduce the operators $O_{\alpha \mathrm{J}}$, defined as

$$
O_{\alpha} \equiv\left(\mathbf{0}, O_{\emptyset}\right)=\sqrt{4 \pi} \sum_{\mathrm{J}} i^{-\mathrm{J}} \sqrt{2 \mathrm{~J}+1} \mathrm{O}_{\alpha \mathrm{J}} .
$$

Thus,

$$
\begin{aligned}
& \mathrm{O}_{\emptyset \mathrm{J}}=j_{\mathrm{J}}(\rho) Y_{\mathrm{J} 0}(\hat{\mathbf{r}}) J_{\emptyset}, \\
& \mathrm{O}_{m \mathrm{~J}}=\sum_{\mathrm{L}} i^{\mathrm{J}-\mathrm{L}} F_{m \mathrm{LJ}} j_{\mathrm{L}}(\rho)\left[Y_{\mathrm{L}}(\hat{\mathbf{r}}) \otimes \mathbf{J}\right]_{\mathrm{J}},
\end{aligned}
$$

where the geometrical factors

$F_{m \mathrm{JL}} \equiv(-)^{m+\mathrm{J}} \sqrt{(2 \mathrm{~L}+1)}\left(\begin{array}{ccc}\mathrm{L} & 1 & \mathrm{~J} \\ 0 & -m & m\end{array}\right)$,

are listed in Table I of Ref. [23].

Explicitly, from (2.7)

$$
\begin{aligned}
O_{\emptyset \mathrm{J}}= & g_{V} \mathcal{M}_{\mathrm{J}}^{V}+i g_{A} \mathcal{M}_{\mathrm{J}}^{A}+i\left(\bar{g}_{\mathrm{A}}+\bar{g}_{\mathrm{P} 1}\right) \mathcal{M}_{0 \mathrm{~J}}^{A}, \\
O_{m \mathrm{~J}}= & i\left(\delta_{m 0} \bar{g}_{\mathrm{P} 2}-g_{\mathrm{A}}+m \bar{g}_{\mathrm{W}}\right) \mathcal{M}_{m \mathrm{~J}}^{A} \\
& +g_{V} \mathcal{M}_{m \mathrm{~J}}^{V}-\delta_{m 0} \bar{g}_{\mathrm{V}} \mathcal{M}_{\mathrm{J}}^{V} .
\end{aligned}
$$

The elementary operators are given by

$$
\begin{aligned}
& \mathcal{M}_{\mathrm{J}}^{V}=j_{\mathrm{J}}(\rho) Y_{\mathrm{J}}(\hat{\mathbf{r}}), \\
& \mathcal{M}_{\mathrm{J}}^{A}=\mathrm{M}^{-1} j_{\mathrm{J}}(\rho) Y_{\mathrm{J}}(\hat{\mathbf{r}})(\boldsymbol{\sigma} \cdot \nabla), \\
& \mathcal{M}_{m \mathrm{~J}}^{A}=\sum_{\mathrm{L} \geqslant 0} i^{\mathrm{J}-\mathrm{L}-1} \mathcal{F}_{m \mathrm{LJ}} j_{\mathrm{L}}(\rho)\left[Y_{\mathrm{L}}(\hat{\mathbf{r}}) \otimes \boldsymbol{\sigma}\right]_{\mathrm{J}}, \\
& \mathcal{M}_{m \mathrm{~J}}^{V}=\mathrm{M}^{-1} \sum_{\mathrm{L} \geqslant 0} i^{\mathrm{J}-\mathrm{L}-1} F_{m \mathrm{LJ}} j_{\mathrm{L}}(\rho)\left[Y_{\mathrm{L}}(\hat{\mathbf{r}}) \otimes \nabla\right]_{\mathrm{J}} .
\end{aligned}
$$


Here we make use of the conserved vector current (CVC). From (2.14), (2.15), and [46, Eqs. (10.45) and (9.7)]

$\mathbf{k} \cdot \mathbf{0}^{V}=\kappa O_{0}^{V}=k_{\emptyset} O_{\emptyset}^{V}$

which yields

$g_{V} \mathcal{M}_{0 \mathrm{~J}}^{V}-\bar{g}_{\mathrm{V}} \mathcal{M}_{\mathrm{J}}^{V}=\frac{k_{\emptyset}}{\kappa} g_{V} \mathcal{M}_{\mathrm{J}}^{V}$.

Therefore, from (2.15)

$$
\begin{aligned}
\mathrm{O}_{m \mathrm{~J}}= & i\left(\delta_{m 0} \bar{g}_{\mathrm{P} 2}-g_{\mathrm{A}}+m \bar{g}_{\mathrm{W}}\right) \mathcal{M}_{m \mathrm{~J}}^{A} \\
& +2|m| \bar{g}_{\mathrm{V}} \mathcal{M}_{m \mathrm{~J}}^{V}+\delta_{m 0} \frac{k_{\emptyset}}{\kappa} g_{\mathrm{V}} \mathcal{M}_{\mathrm{J}}^{V} .
\end{aligned}
$$

The elementary operators $\mathcal{M}_{\mathrm{J}}^{V}, \mathcal{M}_{\mathrm{J}}^{A}, \mathcal{M}_{0 \mathrm{~J}}^{A}$ and $\mathcal{M}_{0 \mathrm{~J}}^{V}$ are real, but $\mathcal{M}_{ \pm 1 \mathrm{~J}}^{A}$ and $\mathcal{M}_{ \pm 1 \mathrm{~J}}^{V}$ are not, and it is convenient to put in evidence their real and imaginary parts, expressing them as

$\mathcal{M}_{ \pm 1 \mathrm{~J}}=\mathcal{M}_{1 \mathrm{~J}}^{R} \pm i \mathcal{M}_{1 \mathrm{~J}}^{I}$

with $\mathcal{M}_{1 \mathrm{~J}}^{R}$, and $\mathcal{M}_{1 \mathrm{~J}}^{I}$ arising, respectively, from the terms in (2.16) with $L=J \pm 1$, and $L=J$. Note that $F_{ \pm 1 \mathrm{JJ}}=\mp 1 / \sqrt{2}$.

It is also convenient to separate the elementary operators into:

- natural parity (NP) $\left(\pi=(-)^{J}\right): \mathcal{M}_{J}^{V}, \mathcal{M}_{1 j}^{A, I}$, and $\mathcal{M}_{1 \mathrm{j}}^{V, R}$, and

- unnatural parity (UP) $\left(\pi=(-)^{\mathrm{J}+1}\right): \mathcal{M}_{\jmath}^{A}, \mathcal{M}_{1 \mathrm{~J}}^{V, I}, \mathcal{M}_{0 \mathrm{~J}}^{A}$, and $\mathcal{M}_{1 \mathrm{~J}}^{A, R}$.

The operators $\mathrm{O}_{\alpha \mathrm{J}} \equiv\left(\mathrm{O}_{\emptyset \mathrm{J}}, \mathrm{O}_{m \mathrm{~J}}\right)$ can be express as a sum of real and imaginary operators, i.e., $\mathrm{O}_{\alpha \mathrm{J}}=\mathrm{O}_{\alpha \mathrm{J}}^{R}+i \mathrm{O}_{\alpha \mathrm{J}}^{I}$, with $\mathrm{O}_{\alpha \mathrm{J}}^{R}\left(\mathrm{O}_{\alpha \mathrm{J}}^{I}\right)$ being an NP (UP) operator. This is a very important finding because it implies that $\mathrm{O}_{\alpha \mathrm{J}}^{R}$ and $\mathrm{O}_{\alpha \mathrm{J}}^{I}$ do not contribute simultaneously, and, therefore, one always can deal only with real operators.

In summary, natural and unnatural parity operators are, respectively:

$\mathrm{O}_{\emptyset \mathrm{J}}^{R}=g_{V} \mathcal{M}_{\mathrm{J}}^{V}$

$\mathrm{O}_{0 \mathrm{~J}}^{R}=\frac{k_{\emptyset}}{\kappa} g_{\mathrm{V}} \mathcal{M}_{\mathrm{J}}^{V}$,

$\mathrm{O}_{m \neq 0 \mathrm{~J}}^{R}=\left(m g_{\mathrm{A}}-\bar{g}_{\mathrm{W}}\right) \mathcal{M}_{1 \mathrm{~J}}^{A, I}+g_{V} \mathcal{M}_{1 \mathrm{~J}}^{V, R}$,

and

$\mathrm{O}_{\emptyset \mathrm{J}}^{I}=g_{A} \mathcal{M}_{\mathrm{J}}^{A}+\left(\bar{g}_{\mathrm{A}}+\bar{g}_{\mathrm{P} 1}\right) \mathcal{M}_{0 \mathrm{~J}}^{A}$,

$\mathrm{O}_{0 J}^{I}=\left(\bar{g}_{\mathrm{P} 2}-g_{\mathrm{A}}\right) \mathcal{M}_{0 J}^{A}$,

$\mathrm{O}_{m \neq 0 \mathrm{~J}}^{I}=\left(-g_{\mathrm{A}}+m \bar{g}_{\mathrm{W}}\right) \mathcal{M}_{1 \mathrm{~J}}^{A, R}+g_{V} \mathcal{M}_{1 \mathrm{~J}}^{V, I}$.

\subsection{Neutrino-nucleus cross section}

For the neutrino-nucleus reaction, the momentum transfer is $k=p_{\ell}-q_{v}$, and the corresponding cross section reads

$\sigma\left(E_{\ell}, J_{f}\right)=\frac{\left|\mathbf{p}_{\ell}\right| E_{\ell}}{2 \pi} F\left(Z \pm 1, E_{\ell}\right) \int_{-1}^{1} d(\cos \theta) \mathcal{T}_{\sigma}\left(\mathrm{q}, J_{f}\right)$,

where $F\left(Z \pm 1, E_{\ell}\right)$ is the Fermi function $(Z+1$, for neutrino, and $Z-1$, for antineutrino), $\theta \equiv \hat{\mathbf{q}} \cdot \hat{\mathbf{p}}$ is the angle between the incident neutrino and ejected lepton, and the transition amplitude is

$\mathcal{T}_{\sigma}\left(\kappa, J_{f}\right)=\frac{1}{2 J_{i}+1} \sum_{s_{\ell}, s_{v}} \sum_{M_{i}, M_{f}}\left|\left\langle J_{f} M_{f}\left|H_{W}\right| J_{i} M_{i}\right\rangle\right|^{2}$.
After expressing the spatial part of the lepton traces $\mathcal{L}_{\alpha \beta}$ in spherical coordinates, and applying the Wigner-Eckart theorem, one can cast the transition amplitude in the compact form [23]

$$
\begin{aligned}
\mathcal{T}_{\sigma}\left(\kappa, J_{f}\right)= & \frac{4 \pi G^{2}}{2 J_{i}+1} \sum_{J}\left[\left|\left\langle J_{f} \| \mathrm{O}_{\emptyset \jmath}|| J_{i}\right\rangle\right|^{2} \mathcal{L}_{\emptyset}\right. \\
& +\sum_{m=0 \pm 1}\left|\left\langle J_{f}|| O_{m J} \| J_{i}\right\rangle\right|^{2} \mathcal{L}_{m} \\
& \left.-2 \Re\left(\left\langle J_{f} \| \mathrm{O}_{\emptyset \jmath}|| J_{i}\right\rangle\left\langle J_{f}\left\|\mathrm{O}_{0 J}\right\| J_{i}\right\rangle\right) \mathcal{L}_{\emptyset 0}\right] .
\end{aligned}
$$

The explicit expressions for the traces $\mathcal{L}_{\emptyset} \equiv \mathcal{L}_{\emptyset \emptyset}, \mathcal{L}_{m} \equiv \mathcal{L}_{m m}$, and $\mathcal{L}_{\emptyset 0}$ are [23]

$\mathcal{L}_{\emptyset \emptyset}=1+\frac{|\mathbf{p}| \cos \theta}{E_{\ell}}$,

$\mathcal{L}_{\emptyset 0}=\left(\frac{q_{0}}{E_{v}}+\frac{p_{0}}{E_{\ell}}\right)$,

$\mathcal{L}_{0}=1+\frac{2 q_{0} p_{0}}{E_{\ell} E_{v}}-\frac{|\mathbf{p}| \cos \theta}{E_{\ell}}$,

$\mathcal{L}_{ \pm 1}=1-\frac{q_{0} p_{0}}{E_{\ell} E_{v}} \pm\left(\frac{q_{0}}{E_{v}}-\frac{p_{0}}{E_{\ell}}\right) S_{1}$,

with

$q_{0}=\hat{k} \cdot \mathbf{q}=\frac{E_{v}\left(|\mathbf{p}| \cos \theta-E_{v}\right)}{\kappa}$,

$p_{0}=\hat{k} \cdot \mathbf{p}=\frac{|\mathbf{p}|\left(|\mathbf{p}|-E_{\nu} \cos \theta\right)}{\kappa}$,

being the $z$-components of the neutrino and lepton momenta, and $S_{1}= \pm 1$ for NS and AS, respectively.

\section{2. - -Capture rates}

The muon capture transition amplitude $\mathcal{T}_{M C}\left(J_{f}\right)$ can be derived from the result (2.25) for the neutrino-nucleus reaction amplitude, by keeping in mind that: i) the roles of $p$ and $q$ are interchanged within the matrix elements of the leptonic current, which makes that in (2.26) $S_{1} \rightarrow-1$, ii) the momentum transfer turns out to be $k=q-p$, and therefore the signs on the right-hand sides of $\left(q_{0}, p_{0}\right)$ have to be changed, and iii) the threshold values $(\mathbf{p} \rightarrow 0$ : $\left.\mathbf{q} \rightarrow \mathbf{k}, k_{\emptyset} \rightarrow E_{v}-\mathrm{m}_{\ell}\right)$ must be used for the lepton traces. All this yields $q_{0}=E_{v}, p_{0}=0$, and

$\mathcal{L}_{\emptyset \emptyset}=\mathcal{L}_{\emptyset 0}=\mathcal{L}_{0}=1, \quad \mathcal{L}_{1}=0, \quad \mathcal{L}_{-1}=2$.

Instead of summing over the initial lepton spins $s_{\ell}$, as done in (2.24), one has now to average over the same quantum number. We get

$\Lambda\left(J_{f}\right)=\frac{E_{v}^{2}}{2 \pi}\left|\phi_{1 S}\right|^{2} \mathcal{T}_{M C}\left(J_{f}\right)$,

where $\phi_{1 S}$ is the muonic bound state wave function evaluated at the origin, and $E_{v}=\mathrm{m}_{\mu}-\left(\mathrm{M}_{n}-\mathrm{M}_{p}\right)-E_{B}^{\mu}-E_{f}+E_{i}$, where $E_{B}^{\mu}$ is the binding energy of the muon in the $1 S$ orbit. Thus from (2.25) and (2.28)

$$
\begin{aligned}
\mathcal{T}_{\sigma}\left(\kappa, J_{f}\right)= & \frac{4 \pi G^{2}}{2 J_{i}+1} \sum_{J}\left[\left|\left\langle J_{f}\left\|\mathrm{O}_{\emptyset \mathrm{J}}-\mathrm{O}_{0 \mathrm{~J}}\right\| J_{i}\right\rangle\right|^{2}\right. \\
& \left.+2\left|\left\langle J_{f}\left\|\mathrm{O}_{-1 \mathrm{~J}}\right\| J_{i}\right\rangle\right|^{2}\right] .
\end{aligned}
$$

In the case of MC it is convenient to rewrite the effective coupling constants (2.8) as 
$\bar{g}_{\mathrm{V}}=g_{\mathrm{V}} \frac{E_{v}}{2 \mathrm{M}} ; \quad \bar{g}_{\mathrm{A}}=g_{\mathrm{A}} \frac{E_{v}}{2 \mathrm{M}} ;$

$\bar{g}_{\mathrm{W}}=\left(g_{\mathrm{V}}+g_{\mathrm{M}}\right) \frac{E_{v}}{2 \mathrm{M}} ; \quad \bar{g}_{\mathrm{P}}=g_{\mathrm{P}} \frac{E_{v}}{2 \mathrm{M}}$,

where $\bar{g}_{\mathrm{P}}=\bar{g}_{\mathrm{P} 2}-\bar{g}_{\mathrm{P} 1}$. $^{2}$

Thus, natural and unnatural parity operators are now, respectively:

$\mathrm{O}_{\emptyset \mathrm{J}}^{R}-\mathrm{O}_{0 \mathrm{~J}}^{R}=\left(g_{\mathrm{V}}-\frac{k_{\emptyset}}{\kappa} g_{\mathrm{V}}\right) \mathcal{M}_{\mathrm{J}}^{V}=g_{\mathrm{V}} \frac{\mathrm{m}_{\mu}}{E_{v}} \mathcal{M}_{\mathrm{J}}^{V}$,

$\mathrm{O}_{-1 \mathrm{~J}}^{R}=-\left(g_{\mathrm{A}}+\bar{g}_{\mathrm{W}}\right) \mathcal{M}_{1 \mathrm{~J}}^{A, I}+g_{V} \mathcal{M}_{1 \mathrm{~J}}^{V, R}$,

and

$\mathrm{O}_{\emptyset \mathrm{J}}^{I}-\mathrm{O}_{0 \mathrm{~J}}^{I}=g_{A} \mathcal{M}_{\mathrm{J}}^{A}+\left(g_{\mathrm{A}}+\bar{g}_{\mathrm{A}}-\bar{g}_{\mathrm{P}}\right) \mathcal{M}_{0 \mathrm{~J}}^{A}$,

$\mathrm{O}_{-1 \mathrm{~J}}^{I}=-\left(g_{\mathrm{A}}+\bar{g}_{\mathrm{W}}\right) \mathcal{M}_{1 \mathrm{~J}}^{A, R}-g_{V} \mathcal{M}_{1 \mathrm{~J}}^{V, I}$.

\section{Nuclear structure calculation}

\section{1. $P Q R P A$}

The PQRPA for charge-exchange excitations was derived from the time-dependent variational principle in Ref. [29]. In the same reference is also described in details the projected Barden-CooperSchiffer (PBCS) approximation. Basically one employs the number projection operators $\hat{P}_{\mathcal{N}}$ on the $|B C S\rangle$ state. That is: $\hat{P}_{0}=\hat{P}_{Z} \hat{P}_{N}$ for a ground state with $Z$ protons and $N$ neutrons, and $\hat{P}_{\mu}=$ $\hat{P}_{Z+\mu} \hat{P}_{N-\mu}$, with $\mu= \pm 1$, for excited states in nuclei with $Z+\mu$ protons and $N-\mu$ neutrons. In this section we give a brief description of both the PBCS and PQRPA approximations.

The PBCS gap equations are

$2 \bar{e}_{k} u_{k} v_{k}-\Delta_{k}\left(u_{k}^{2}-v_{k}^{2}\right)=0$,

where

$\Delta_{k}=-\frac{1}{2} \sum_{k^{\prime}} \frac{\left(2 j_{k^{\prime}}+1\right)^{1 / 2}}{\left(2 j_{k}+1\right)^{1 / 2}} u_{k^{\prime}} v_{k^{\prime}} \mathrm{G}\left(k k k^{\prime} k^{\prime} ; 0\right) \frac{I^{Z-2}\left(k k^{\prime}\right)}{I^{Z}}$,

are the pairing gaps, and

$$
\begin{aligned}
\bar{e}_{k}= & e_{k} \frac{I^{Z-2}(k)}{I^{Z}}+\sum_{k^{\prime}} \frac{\left(2 j_{k^{\prime}}+1\right)^{1 / 2}}{\left(2 j_{k}+1\right)^{1 / 2}} v_{k^{\prime}}^{2} \\
& \times \mathrm{F}\left(k k k^{\prime} k^{\prime} ; 0\right) \frac{I^{Z-4}\left(k k^{\prime}\right)}{I^{Z}}+\Delta e_{k},
\end{aligned}
$$

are the dressed single-particle energies, where

$$
\begin{aligned}
I^{K}\left(k_{1} k_{2} \cdots k_{n}\right)= & \frac{1}{2 \pi i} \oint \frac{d z}{z^{K+1}} \sigma_{k_{1}} \cdots \sigma_{k_{n}} \\
& \times \prod_{k}\left(u_{k}^{2}+z^{2} v_{k}^{2}\right)^{j_{k}+1 / 2} ;
\end{aligned}
$$

$\sigma_{k}^{-1}=u_{k}^{2}+z_{k}^{2} v_{k}^{2}$

are the PBCS number projection integrals. The PBCS correction term $\Delta e_{k}$ can be found in Ref. [29], G $\left(k k k^{\prime} k^{\prime} ; 0\right)$, and $\mathrm{F}\left(k k k^{\prime} k^{\prime} ; 0\right)$ stand for the usual proton or neutron particle-particle (pp), and particle-hole (ph) matrix elements of the residual interaction $V$, i.e.,

$G(k l m n ; J)=\langle k l ; J|V| m n ; J\rangle$,

$F(k l m n ; J)=\left\langle k l^{-1} ; J|V| m n^{-1} ; J\right\rangle$.

\footnotetext{
2 Note that there is a misprint in Eq. (2.41) of Ref. [23]. Also in Eq. (2.42) of the same reference $\bar{g}_{\mathrm{P} 1}$ should read $\bar{g}_{\mathrm{P}}$.
}

Note that these relations are valid for both identical and nonidentical particles.

The forward-going $\left(X_{\mu}\right)$, and backward-going $\left(Y_{\mu}\right)$ PQRPA amplitudes are obtained by solving the RPA equations

$\left(\begin{array}{cc}A_{\mu} & B \\ -B^{*} & -A_{-\mu}^{*}\end{array}\right)\left(\begin{array}{c}X_{\mu} \\ Y_{\mu}\end{array}\right)=\omega_{\mu}\left(\begin{array}{c}X_{\mu} \\ Y_{\mu}\end{array}\right)$

with the PQRPA matrices defined as:

$$
\begin{aligned}
A_{\mu}( & \left.p n, p^{\prime} n^{\prime} ; J\right) \\
= & \omega_{\mu}^{0} \delta_{p n, p^{\prime} n^{\prime}}+N_{\mu}^{-1 / 2}(p n) N_{\mu}^{-1 / 2}\left(p^{\prime} n^{\prime}\right) \\
& \times\left\{\left[u_{p} v_{n} u_{p^{\prime}} v_{n^{\prime}} I^{Z-1+\mu}\left(p p^{\prime}\right) I^{N-3-\mu}\left(n n^{\prime}\right)\right.\right. \\
& \left.+v_{p} u_{n} v_{p^{\prime}} u_{n^{\prime}} I^{Z-3+\mu}\left(p p^{\prime}\right) I^{N-1-\mu}\left(n n^{\prime}\right)\right] \mathrm{F}\left(p n, p^{\prime} n^{\prime} ; J\right) \\
& +\left[u_{p} u_{n} u_{p^{\prime}} u_{n^{\prime}} I^{Z-1+\mu}\left(p p^{\prime}\right) I^{N-1-\mu}\left(n n^{\prime}\right)\right. \\
& \left.\left.+v_{p} v_{n} v_{p^{\prime}} v_{n^{\prime}} I^{Z-3+\mu}\left(p p^{\prime}\right) I^{N-3-\mu}\left(n n^{\prime}\right)\right] \mathrm{G}\left(p n, p^{\prime} n^{\prime} ; J\right)\right\}, \\
B\left(p n, p^{\prime} n^{\prime} ; J\right) & \\
= & N_{\mu}^{-1 / 2}(p n) N_{-\mu}^{-1 / 2}\left(p^{\prime} n^{\prime}\right) I^{Z-2}\left(p p^{\prime}\right) I^{N-2}\left(n n^{\prime}\right) \\
& \times\left[\left(v_{p} u_{n} u_{p^{\prime}} v_{n^{\prime}}+u_{p} v_{n} v_{p^{\prime}} u_{n^{\prime}}\right) \mathrm{F}\left(p n, p^{\prime} n^{\prime} ; J\right)\right. \\
& \left.+\left(u_{p} u_{n} v_{p^{\prime}} v_{n^{\prime}}+v_{p} v_{n} u_{p^{\prime}} u_{n^{\prime}}\right) \mathrm{G}\left(p n, p^{\prime} n^{\prime} ; J\right)\right],
\end{aligned}
$$

where

$\omega_{\mu}^{0}=\varepsilon_{p}^{Z-1+\mu}+\varepsilon_{n}^{N-1-\mu}$,

are the unperturbed energies,

$N_{\mu}(p n)=I^{Z-1+\mu}(p) I^{N-1-\mu}(n)$,

are the norms,

$\varepsilon_{k}^{K}=\frac{R_{0}^{K}(k)+R_{11}^{K}(k k)}{I^{K}(k)}-\frac{R_{0}^{K}}{I^{K}}$,

are the projected quasiparticle energies, and the quantities $R^{K}$ are defined as [29]

$$
\begin{aligned}
R_{0}^{K}(k)= & \sum_{k_{1}}\left(2 j_{k_{1}}+1\right) v_{k_{1}}^{2} e_{k_{1}} I^{K-2}\left(k k_{1}\right) \\
+ & \frac{1}{4} \sum_{k_{1} k_{2}}\left(2 j_{k_{1}}+1\right)^{1 / 2}\left(2 j_{k_{2}}+1\right)^{1 / 2} \\
& \times\left[v_{k_{1}}^{2} v_{k_{2}}^{2} \mathrm{~F}\left(k_{1} k_{1} k_{2} k_{2} ; 0\right) I^{K-4}\left(k_{1} k_{2} k\right)\right. \\
+ & \left.u_{k_{1}} v_{k_{1}} u_{k_{2}} v_{k_{2}} \mathrm{G}\left(k_{1} k_{1} k_{2} k_{2} ; 0\right) I^{K-2}\left(k_{1} k_{2} k\right)\right], \\
R_{11}^{K}(k k)= & e_{k}\left[u_{k}^{2} I^{K}(k k)-v_{k}^{2} I^{K-2}(k k)\right] \\
& +\sum_{k_{1}} \frac{\left(2 j_{k_{1}}+1\right)^{1 / 2}}{\left(2 j_{k}+1\right)^{1 / 2}}\left\{v_{k_{1}}^{2} \mathrm{~F}\left(k_{1} k_{1} k k ; 0\right)\right. \\
& \times\left[u_{k}^{2} I^{K-2}\left(k_{1} k k\right)-v_{k}^{2} I^{K-4}\left(k_{1} k k\right)\right] \\
& \left.-u_{k_{1}} v_{k_{1}} u_{k} v_{k} \mathrm{G}\left(k_{1} k_{1} k k ; 0\right) I^{K-2}\left(k_{1} k k\right)\right\} .
\end{aligned}
$$

Both positive and negative solutions are physically meaningful. For $\mu= \pm 1$ the positive solutions describe excitations in the $(Z \pm$ $1, N \mp 1)$ nuclei, while the negative energy solutions represent the positive energy excitations in the $(Z \mp 1, N \pm 1)$. Thus only one RPA equation has to be solved, either for $\mu=+1$, or for $\mu=-1$, to describe the excitations to the $Z \pm 1, N \mp 1$ nuclei. This is wellknown feature of the charge-exchange modes [47-50].

Let us be more specific, and take advantage of the index $f$ to label different final states $\left|J_{f}^{\pi}\right\rangle$ with same spin and parity. Evidently, 
$f$ will run from 1 up the total number $f_{\max }$ of two-quasiparticle configurations $\left|p n J^{\pi}\right\rangle$. Moreover, the eigenvalue problem (3.6) has $2 f_{\max }$ solutions, and we will use the index $F$ to label them. Thus, if $\mu=+1$ one has:

- for $\omega_{+1}\left(J_{F}\right)>0\left(1<F \leqslant f_{\max }\right)$ :

$$
\begin{aligned}
& \omega_{+1}\left(J_{f}\right)=\omega_{+1}\left(J_{F}\right), \\
& X_{+1}\left(p n J_{f}\right)=X_{+1}\left(p n J_{F}\right), \\
& Y_{+1}\left(p n J_{f}\right)=Y_{+1}\left(p n J_{F}\right) ;
\end{aligned}
$$

- for $\omega_{+1}\left(J_{F}\right)<0\left(f_{\max }<F \leqslant 2 f_{\max }\right)$ :

$$
\begin{aligned}
& \omega_{-1}\left(J_{f}\right)=-\omega_{+1}\left(J_{F}\right), \\
& X_{-1}\left(p n J_{f}\right)=Y_{+1}^{*}\left(p n J_{F}\right), \\
& Y_{-1}\left(p n J_{f}\right)=X_{+1}^{*}\left(p n J_{F}\right) .
\end{aligned}
$$

Finally, to store the eigenvalues and eigenfunctions it is convenient to define the index $F$ as

$F= \begin{cases}f, & \text { for } F \leqslant f_{\max } \\ 2 f_{\max }-f+1, & \text { for } F>f_{\max }\end{cases}$

\subsection{QRPA}

The usual gap equations are obtained from Eqs. (3.6)-(3.7) by:

1. Making the replacement $e_{k} \rightarrow e_{k}-\lambda_{k}$, with $\lambda_{k}$ being the chemical potential, and taking the limit $I^{K} \rightarrow 1$. That is, Eq. (3.1) remains as it is, but instead of (3.2) and (3.3) one has now

$\Delta_{k}=-\frac{1}{2} \sum_{k^{\prime}} \frac{\left(2 j_{k^{\prime}}+1\right)^{1 / 2}}{\left(2 j_{k}+1\right)^{1 / 2}} u_{k^{\prime}} v_{k^{\prime}} \mathrm{G}\left(k k k^{\prime} k^{\prime} ; 0\right)$,

and

$\bar{e}_{k}=e_{k}-\lambda_{k}+\sum_{k^{\prime}} \frac{\left(2 j_{k^{\prime}}+1\right)^{1 / 2}}{\left(2 j_{k}+1\right)^{1 / 2}} v_{k^{\prime}}^{2} \mathrm{~F}\left(k k k^{\prime} k^{\prime} ; 0\right)$.

2. Impose the subsidiary conditions

$Z=\sum_{j_{p}}\left(2 j_{p}+1\right)^{2} v_{j_{p}}^{2}, \quad N=\sum_{j_{n}}\left(2 j_{n}+1\right)^{2} v_{j_{n}}^{2}$,

as the number of particles is not any more a good quantum number.

In this way the usual BCS gap equations read

$2\left(e_{k}-\lambda_{t}\right) u_{k} v_{k}=\left(u_{k}^{2}-v_{k}^{2}\right) \Delta_{k}$.

This equation, together with the normalization condition $u_{k}^{2}+v_{k}^{2}$ $=1$, has as solution the occupation probabilities (for example, from Chapter I of Rowe [47])

$u_{k}^{2}=\frac{1}{2}\left(1+\frac{e_{k}-\lambda_{k}}{E_{k}}\right), \quad v_{k}^{2}=\frac{1}{2}\left(1-\frac{e_{k}-\lambda_{k}}{E_{k}}\right)$,

which depend on the quasiparticle energies

$E_{k}=\sqrt{\left(e_{k}-\lambda_{k}\right)^{2}+\Delta_{k}^{2}}$,

and the pairing gaps

$\Delta_{k}=-\frac{1}{2} \sum_{k^{\prime}} \frac{\left(2 j_{k^{\prime}}+1\right)^{1 / 2}}{\left(2 j_{k}+1\right)^{1 / 2}} u_{k^{\prime}} v_{k^{\prime}} \mathrm{G}\left(k k k^{\prime} k^{\prime} ; 0\right)$.
The QRPA equations are recovered from (3.6) by i) dropping the index $\mu$, ii) taking the limit $I^{K} \rightarrow 1$, and iii) substituting the unperturbed PBCS energies by the BCS energies relative to the Fermi level, defined by equation

$E_{k}^{( \pm)}= \pm E_{k}+\lambda_{k}$

where $E_{k}$ are the usual BCS quasiparticle energies defined in (3.20). In this way the unperturbed energies in (3.7) are replaced by

$\omega_{\mu}^{0}=E_{j_{p}}+E_{j_{n}}+\mu\left(\lambda_{\mathrm{p}}-\lambda_{\mathrm{n}}\right)$.

These energies, however, are not used in the QRPA eigenvalue problem. Namely, the coefficients $X\left(p n J_{f}\right)$ and $Y\left(p n J_{f}\right)$, and the eigenvalues $\omega\left(J_{f}\right)$ are obtained from

$\left(\begin{array}{ll}\mathcal{A} & \mathcal{B} \\ \mathcal{B} & \mathcal{A}\end{array}\right)\left(\begin{array}{c}X \\ Y\end{array}\right)=\omega\left(\begin{array}{c}X \\ -Y\end{array}\right)$

where

$$
\begin{aligned}
\mathcal{A}\left(p n p^{\prime} n^{\prime} ; J\right)= & \left(E_{p}+E_{n}\right) \delta_{p p^{\prime}} \delta_{n n^{\prime}} \\
& +\left(u_{p} v_{n} u_{p^{\prime}} v_{n^{\prime}}+v_{p} u_{n} v_{p^{\prime}} u_{n^{\prime}}\right) F\left(p n p^{\prime} n^{\prime} ; J\right) \\
& +\left(u_{p} u_{n} u_{p^{\prime}} u_{n^{\prime}}+v_{p} v_{n} v_{p^{\prime}} v_{n^{\prime}}\right) G\left(p n p^{\prime} n^{\prime} ; J\right), \\
\mathcal{B}\left(p n p^{\prime} n^{\prime} ; J\right)= & \left(v_{p} u_{n} u_{p^{\prime}} v_{n^{\prime}}+u_{p} v_{n} v_{p^{\prime}} u_{n^{\prime}}\right) F\left(p n p^{\prime} n^{\prime} ; J\right) \\
& +\left(u_{p} u_{n} v_{p^{\prime}} v_{n^{\prime}}+v_{p} v_{n} u_{p^{\prime}} u_{n^{\prime}}\right) G\left(p n p^{\prime} n^{\prime} ; J\right) .
\end{aligned}
$$

In the pn-QRPA the eigenvalues occur in pairs $\pm \omega\left(J_{f}\right)$, but the negative energies don't have a direct physical meaning. The perturbed energies for daughter $(Z+\mu, N-\mu)$ nuclei are defined as

$\omega_{\mu}\left(J_{f}\right)=\omega\left(J_{f}\right)+\mu\left(\lambda_{p}-\lambda_{n}\right)$.

There is, however, only one set of eigenfunctions $\left(X\left(p n J_{f}\right)\right.$, $Y\left(p n J_{f}\right)$ ) for both $\mu=1$, and $\mu=-1$. This is a very important difference in relation to the PQRPA case, which is crucial for the distribution of the transition strengths.

\subsection{Nuclear matrix elements}

When the excited states $\left|J_{f}\right\rangle$ in the final $(Z \pm 1, N \mp 1)$ nuclei are described within the PQRPA, the transition amplitudes for the multipole charge-exchange operators (2.21) and (2.22) read

$$
\begin{aligned}
& \left\langle J_{f}, Z+\mu, N-\mu\left\|O_{J}\right\| 0^{+}\right\rangle \\
& =\frac{1}{\left(I^{Z} I^{N}\right)^{1 / 2}} \sum_{p n}\left[\frac{\Lambda_{\mu}(p n J)}{\left(I^{Z-1+\mu}(p) I^{N-1+\mu}(n)\right)^{1 / 2}} X_{\mu}^{*}\left(p n J_{f}\right)\right. \\
& \left.\quad+\frac{\Lambda_{-\mu}(p n J)}{\left(I^{Z-1-\mu}(p) I^{N-1-\mu}(n)\right)^{1 / 2}} Y_{\mu}^{*}\left(p n J_{f}\right)\right],
\end{aligned}
$$

with the one-body matrix elements given by

$\Lambda_{\mu}(p n J)=-\frac{\left\langle p\left\|O_{J}\right\| n\right\rangle}{\sqrt{2 J+1}} \begin{cases}u_{\mathrm{p}} v_{\mathrm{n}}, & \text { for } \mu=+1, \\ u_{\mathrm{n}} v_{\mathrm{p}}, & \text { for } \mu=-1,\end{cases}$

and $J=\mathrm{J}$.

In the QRPA case, using the limit $I^{K} \rightarrow 1$ in (3.27), the nuclear matrix elements for the multipole charge-exchange operators $O_{J}$ are

$$
\begin{aligned}
& \left\langle J_{f}, Z+\mu, N-\mu\left\|\mathrm{O}_{J}\right\| 0^{+}\right\rangle \\
& \quad=\sum_{p n}\left[\Lambda_{\mu}(p n J) X^{*}\left(p n J_{f}\right)+\Lambda_{-\mu}(p n J) Y^{*}\left(p n J_{f}\right)\right],
\end{aligned}
$$

with the same one-body matrix elements (3.28). 
The unperturbed and perturbed transition strengths are defined, respectively, as

$S_{\mu}^{0}(p n J)=\left|\Lambda_{\mu}(p n J)\right|^{2}$

and

$S_{\mu}\left(J_{f}\right)=\left|\left\langle J_{f}, Z+\mu, N-\mu\left\|\mathrm{O}_{\mathrm{J}}\right\| 0^{+}\right\rangle\right|^{2}$.

One might be particularly interested in the Gamow-Teller (GT) and Fermi (F) $\beta$-decay strengths (B-values), in which case (3.30) and (3.31) are evaluated for the operators $\widetilde{O}_{J}$, which don't contain the radial form factors $j_{\mathrm{J}}(\rho)$. That is, $\widetilde{\mathrm{O}}_{0}=1$, and $\widetilde{\mathrm{O}}_{1}=\sigma$, for $\mathrm{F}$ and GT operators, respectively. We denote the B-values as $\widetilde{S}_{\mu}(p n J)$ and $\widetilde{\mathcal{S}}_{\mu}\left(J_{f}\right)$. Occasionally one also might want to calculate the energy distribution of the last one, i.e.,

$\widetilde{S}_{\mu}\left(J_{f}, E\right)=\frac{\eta}{\pi} \sum_{f} \frac{\widetilde{S}_{\mu}\left(J_{f}\right)}{\eta^{2}+\left(E-\omega_{J_{f}}\right)^{2}}$,

where one usually takes $\eta=1 \mathrm{MeV}$.

\section{Computer program and user's manual}

The QRAP code evaluates the electron neutrino-nucleus interaction described by Eq. (2.1) (IREAC $=1$ for NS, IREAC $=2$ for AS) and (2.2) (IREAC $=0$ for MC). The processes, from the ground state of the even-even father nucleus $(Z, N)$ to the excited states with spin (ISPIN) and parity (IPARI) in the odd-odd daughter nucleus $(Z \pm 1, N \mp 1)$, are calculated by using the QRPA model $(\mathrm{IQP}=0)$ or the PQRPA (IQP = 1). These options must be setup in the input data file, qrapin.dat, which is supplemented with two included files:

(a) sp.inc, containing the dimensions of single-particle quantum numbers, occupation probabilities, quasiparticle quantities and strength amplitudes for allowed transitions;

(b) conf.inc, which has the dimensions for the quasiparticle state configurations, the Hamiltonian matrices $(A, B)$ or $(\mathcal{A}, \mathcal{B})$ which are diagonalized, the forward and backward amplitudes, and the eigenvalues.

There are two input files: 1) qrapout.dat, where is listed the output file that shows the neutrino/antineutrino $(v / \bar{v})$ cross section, as a function of the incident neutrino, or the muon capture rate, for each state of a given nuclear spin in the daughter nucleus, and 2) the above mentioned qrapin.dat, which contains: a) the quantum numbers of all single-particle state (sps), and the corresponding single particle energies (s.p.e.), b) the mass and the proton number of the parent nucleus, c) the neutron and proton pairing strengths for the BCS approximation, d) the particle-particle, and particle-hole strengths of the residual interaction, e) the position of the Fermi level, and the experimental gap for neutrons and protons, and f) the $Q$-value for the $v / \bar{v}$ scattering.

There are three default output files. Two of them, AUXI.OUT and OUT.OUT, contain the results of the nuclear structure model, whereas the results for the weak processes appear in the file created by qrapout.dat. For example, if one is interested in the multipole $J_{f}^{\pi}=1^{+}$with a single-particle space of six levels in ${ }^{12} \mathrm{C}$ ("set 1"), we can introduce in qrapout.dat the file names QNC.out (PNC.out) for neutrino capture, QAC.out (PAC.out) for antineutrino capture, QMC.out (PMC.out) for muon capture, using the QRPA (PQRPA) model. The auxiliary output files AUXI.OUT and OUT.OUT are relabeled to (QAUXI.OUT, QOUT.OUT) and (PAUXI.OUT, POUT.OUT) for QRPA and PQRPA respectively.

All just mentioned outputs are included as examples. The following units are employed: i) $10^{-42} \mathrm{~cm}^{2}$, for neutrino- or antineutrino-nucleus cross sections, ii) $10^{4} \mathrm{~s}^{-1}$, for muon capture rates, and iii) $\mathrm{MeV}$, for energies.

\subsection{Reading the dato}

There are three sets of input data in qrapin.dat separated in modules labeled as: *Data set 1 for a single-particle space of six levels in ${ }^{12} \mathrm{C}\left(0,1\right.$, and $2 \hbar \omega$ oscillator shells), ${ }^{*}$ Data set 2 for a single-particle space of ten levels in ${ }^{12} \mathrm{C}(0,1,2$, and $3 \hbar \omega$ oscillator shells), and ${ }^{*}$ Data set 3 for single-particle space of 12 levels in ${ }^{56} \mathrm{Fe}(2,3$, and $4 \hbar \omega$ oscillator shells).

For each one of these input data, the number of sps represents the available space where one wants to solve the BCS (or PBCS) problem given by Eqs. (3.17) and (3.18) ((3.1)-(3.2)). It contains the necessary number of harmonic oscillator shells leading to a smooth smearing of the Fermi's surface. The Fermi level with the neighboring levels constitute the active shell for the mentioned smearing. For example, in ${ }^{12} \mathrm{C}$ (ground state with $J=0^{+}$) the active shell is composed by the $1 p_{3 / 2}$ and $1 p_{1 / 2}$ levels. According to the singleparticle shell model the sps filled up the $1 p_{3 / 2}$ orbital, and the nucleons can be promoted to the $1 p_{1 / 2}$, creating a particle state in $1 p_{1 / 2}$ and a hole state in $1 p_{3 / 2}$. This scheme describes the first particle-hole $(\mathrm{ph})$ excitation on ${ }^{12} \mathrm{C}$ in order to obtain the ${ }^{12} \mathrm{~N}$ or ${ }^{12} \mathrm{~B}$ ground state with $J=1^{+}$, by promoting a proton or a neutron, respectively.

Let us show, as example, a data input of six sps for ${ }^{12} \mathrm{C}$ : ${ }^{*}$ Data set 1 . The rows starting with a symbol "*” are not read as input and just serve to remind the user on the meaning of the physical quantities. Taking out the comments "*” in the first lines of this file, we have

$\begin{array}{lrrrccccccccc} & 1 & +1 & 0.0 & 1 & 1 & & & & & & & \\ 06 & & 06 & 0 & & 1 & & 1 & 0 & & & & \\ 101 & -20.09 & 112 & -6.02 & 111 & -0.29 & 123 & 3.07 & 201 & 3.85 & 122 & 7.18 \\ 101 & -18.19 & 112 & -3.17 & 111 & 2.79 & 123 & 5.73 & 201 & 6.06 & 122 & 9.36 \\ 12 & 06 & 28.80 & & 28.85 & 30.0 & 50.0 & 27.0 & 64.0 & \\ 2 & 1 & & 6 & & 6 & 1.00 & 6.88 & & & \\ 2 & 1 & & 6 & & 6 & 1.00 & 7.00 & & & & \end{array}$

First line: Nuclear spin (ISPIN = 1) of the daughter nucleus, parity (IPARI $=+1$ ), coupling strength of particle-particle channel $(t=0.0)$ (see definition below), index of neutrino reaction $(I R E A C=1)$ and the index $(I Q P=1)$ to solve the PQRPA problem.

Second line: Number of neutron sps $(N S N=06)$, number of proton sps (NSP $=06$ ), index to solve the QRPA equation in the Tamm-Damcoff approximation (ITD: 0 no, 1 yes), index to print the matrix elements of the nuclear Hamiltonian to be diagonalized (MAPR: 0 no, 1 yes), index to solve the BCS equation with the selfenergy term (IFMU: 0 no, 1 yes), index to make the QRPA matrix with the branch $(Z+1, N-1)(\mu=1)$ or $(Z-1, N+1)(\mu=-1)$ of PQRPA (IPRO: $0(\mu=1), 1(\mu=-1)$ ).

Third and fourth lines: quantum numbers and the s.p.e. for each neutron and proton sps, respectively. They are represented in the same way as in the shell model scheme, with their respective quantum numbers $\left(n \ell\left(j+\frac{1}{2}\right)\right)$. For example, $101 \rightarrow 1 s_{1 / 2}$ where 1 is principal quantum number, corresponding to the first harmonic oscillator level $(n), 0$ corresponds to the orbital angular momentum $\ell \equiv s$ and the last number $1 \equiv j+\frac{1}{2}=\frac{1}{2}+\frac{1}{2}$. Tables 1 and 2 show the notation and the corresponding quantum numbers, as well as the PBCS quasiparticle energies $e_{j}^{N}$ and $e_{j}^{Z}$, defined by (3.10).

Fifth line: Mass number $A(\mathrm{IAM}=12)$, proton number $Z$ $(I Z=6)$, and the following coupling constants: 1$)$ neutrons and proton pairing $v_{s}^{\text {pair }}(\mathrm{VspairN}=28.80)$, and $v_{s}^{\text {pair } P}(\mathrm{Vspair} \mathrm{P}=$ $28.85), 2)$ singlet and triplet particle-particle $(p p) v_{s}^{p p}(\mathrm{VsPP}=30)$, and $v_{t}^{p p}(\mathrm{VtPP}=50)$, and iii) singlet and triplet particle-hole $(p h)$ $v_{s}^{p h}(\mathrm{VsPH}=27), v_{t}^{p h}(\mathrm{VtPH}=64)$. 
Table 1

Notation for the quantum numbers, the resulting quasiparticle energies $e_{j}^{N}$ for neutrons and $e_{j}^{Z}$ for protons, and the pairing strength $v_{s}^{\text {pair }}$ within the PBCS. The energies are given in units of $\mathrm{MeV}$, and $v_{s}^{\text {pair }}$ is dimensionless.

\begin{tabular}{lllllrr}
\hline Notation & Shell & $n$ & $\ell$ & $j+1 / 2$ & $e_{j}^{N}$ & \multicolumn{1}{c}{$e_{j}^{Z}$} \\
\hline 101 & $1 s_{1 / 2}$ & 1 & 0 & 1 & -20.09 & -18.19 \\
112 & $1 p_{3 / 2}$ & 1 & 1 & 2 & -6.02 & -3.17 \\
111 & $1 p_{1 / 2}$ & 1 & 1 & 1 & -0.29 & 2.79 \\
123 & $1 d_{5 / 2}$ & 1 & 2 & 3 & 3.07 & 5.73 \\
201 & $2 s_{1 / 2}$ & 1 & 0 & 1 & 3.85 & 6.06 \\
122 & $1 d_{3 / 2}$ & 1 & 2 & 2 & 7.18 & 9.36 \\
$v_{s}^{\text {pair }}$ & & & & & 28.80 & 28.85
\end{tabular}

Table 2

Spin and parity for the one-quasiparticle space used in the input for ${ }^{12} \mathrm{C}$.

\begin{tabular}{lllllll}
\hline & $1 s_{1 / 2}$ & $1 p_{3 / 2}$ & $1 p_{1 / 2}$ & $2 s_{1 / 2}$ & $1 d_{5 / 2}$ & $1 d_{3 / 2}$ \\
\hline $1 s_{1 / 2}$ & $0^{+}, 1^{+}$ & & & & & \\
$1 p_{3 / 2}$ & $1^{-}, 2^{-}$ & $0^{+}, 1^{+}$ & & & & \\
& & $2^{+}, 3^{+}$ & & & & \\
$1 p_{1 / 2}$ & $0^{-}, 1^{-}$ & $1^{+}, 2^{+}$ & $0^{+}, 1^{+}$ & & & \\
$2 s_{1 / 2}$ & $0^{+}, 1^{+}$ & $1^{-}, 2^{-}$ & $0^{-}, 1^{-}$ & $0^{+}, 1^{+}$ & & \\
$1 d_{5 / 2}$ & $2^{+}, 3^{+}$ & $1^{-}, 2^{-}$ & $2^{-}, 3^{-}$ & $2^{+}, 3^{+}$ & $0^{+}, 1^{+}, 2^{+}$ & \\
& & $3^{-}, 4^{-}$ & & & $3^{+}, 4^{+}, 5^{+}$ & \\
$1 d_{3 / 2}$ & $1^{+}, 2^{+}$ & $0^{-}, 1^{-}$ & $1^{-}, 2^{-}$ & $1^{+}, 2^{+}$ & $1^{+}, 2^{+}$ & $0^{+}, 1^{+}$ \\
& & $2^{-}, 3^{-}$ & & & $3^{+}, 4^{+}$ & $2^{+}, 3^{+}$
\end{tabular}

Sixth and seventh lines: Position of the Fermi level (LEVEL $=2$ ), initial (IIQ = 1 ) and final (IFQ =6) states for which the BCS equations must be solved, number of particles interacting $(\mathrm{NPIQ}=6)$ in these levels, and the experimental gap (DELTAQ $=6.88$ or 7.00 ) defined below in Eq. (4.2); for neutrons and protons, fifth and sixth lines respectively.

Eighth line: $Q$-value minus the lepton mass for $v / \bar{v}$ scattering $\left(E G S=17.338\right.$ for $\left.{ }^{12} \mathrm{~N}[51]\right)$. It can be fixed as being the energy of the ground state in the daughter nucleus. The lepton mass must be added to EGS to obtain the $Q$-value for the reaction.

\subsection{Running the code}

As first step the QRAP solves the BCS problem. In this case, one needs to adjust the pairing strength to reproduce the experimental pairing gap.

Next, one can solve the PBCS problem or directly the QRPA if the option IQP $=0$ was selected. If IQP $=1$ then the PQRPA equations are solved. It means that QRAP firstly calculates the nuclear matrix elements in the QRPA or PQRPA by selecting the option $\mathrm{IQP}=0$ or 1 , appropriately. The option for which type of weak interaction process one wants to evaluate is adopted with IREAC in the input data. We recommend first to adjust the pairing strength as it is explained below. After this it is convenient to fit the parameters of the residual interaction using the option IREAC $=3$ for the muon capture rate because this calculation is fast. Physically you can check quickly how good is your choice of parameters because the values for inclusive muon-capture rate, and GT B-values are available in the literature (see for example Refs. [52,53]).

For the residual interaction the code assumes a delta force,

$V=-4 \pi\left(v_{s} P_{s}+v_{t} P_{t}\right) \delta(r)$

which has been used extensively in the literature [26-28] to describe single and double beta decays.

Next, we explain how the parameters of the interaction are adjusted using for example the input data for six levels in ${ }^{12} \mathrm{C}$. The results are presented in output file OUT.OUT.
Adjusting the gap $\Delta_{k}$

The parameters $v_{s}^{\text {pair } N}$ and $v_{s}^{\text {pair } P}$ are adjusted to reproduce the experimental gap $\Delta^{N}$ for neutrons, and $\Delta^{Z}$ for protons, by solving the BCS equations (3.17) and (3.18) in a self-consistent way. The experimental gaps, according [54, Eq. (2.96)], are:

$\Delta^{N}=-\frac{1}{2}\{\mathcal{B}(Z, N-1)-2 \mathcal{B}(Z, N)+\mathcal{B}(Z, N+1)\}$,

$\Delta^{Z}=-\frac{1}{2}\{\mathcal{B}(Z-1, N)-2 \mathcal{B}(Z, N)+\mathcal{B}(Z+1, N)\}$,

where $\mathcal{B}(N, Z)$ is the binding energy of the even-even nucleus $(Z, N)$. This is the most common fit (Fit1) used in several works with standard QRPA [28-30]. In this case, the $\Delta^{N(Z)}$ must be equal or approximately equal to the energy $\Delta_{j_{k}=F L}^{N(Z)}$ of the corresponding to the Fermi level (FL). To solve the set of PBCS coupled equations (3.1)-(3.4) for $u_{k}$ and $v_{k}$ it is recommended to obtain first the solutions for the BCS problem, as these probability occupations are use as input for the PBCS case. The PBCS coupled nonlinear equations are solved consistently with Powell Hybrid method using subroutine HYBRD [55].

The results of the BCS or PBCS problem are shown as tables in the first lines of OUT.OUT for neutrons and protons, respectively. The quantities defined by (3.10) and (3.11) are presented there. In particular, the projected quasiparticle energy defined in (3.10) are

PROYSP $= \begin{cases}\mathrm{E}(+)=\varepsilon_{k}^{K}, & \text { with } k \text { above Fermi level, } \\ \mathrm{E}(-)=\varepsilon_{k}^{K-2}, & \text { with } k \text { below Fermi level, }\end{cases}$

which means that $\mathrm{E}(+)$ corresponds to a particle state, and $\mathrm{E}(-)$ to a hole state. The values of $\Delta_{j_{k}}^{N(Z)}$ are shown in the ninth column of the table labeled as CONFIGURATION SPACE. This Fit1 comes from the fact that the experimental energy difference between the states that lie just above ( $p$ state) and just above ( $h$ state) the FL is approximately twice the experimental gap, i.e.,

$E_{p}^{K}-E_{h}^{K} \simeq 2 \Delta^{K}$

for $K=N$ or $Z$.

There is another fitting procedure for the pairing gap that is called by Fit2. In Fit2, all the s.p.e. $e_{j}^{N(Z)}$ from Table 1 are varied with a $\chi^{2}$ search to account for the experimental spectra $E_{j}$ :

$\epsilon_{k}^{Z(N)} \rightarrow E_{p}^{Z(N)} \equiv \mathrm{E}(+), \quad$ for a particle state,

$\epsilon_{k}^{Z-2(N-2)} \rightarrow E_{h}^{Z(N)} \equiv \mathrm{E}(-), \quad$ for a hole state.

In Fit2, Eq. (4.4) is automatically satisfied. This procedure was employed to obtain the $e_{j}$ spectra shown in [23, Table III], whereas the $e_{j}$ for the reduced space of six levels in the present example are shown in Table 1. These s.p.e. are used in the input data Data set 1 .

To make the calculations as simple as possible the Fit1 procedure is the usual choice, with the $e_{j}$ spectra obtained either from a harmonic oscillator or from a Wood-Saxon potential, and by varying the coupling $v_{s}^{\text {pair } N}$ and $v_{s}^{\text {pair } P}$ to satisfy the condition $\Delta_{j_{k}=F L}^{N(Z)} \approx \Delta^{N(Z)}$.

For ${ }^{56} \mathrm{Fe}$ the input data is called ${ }^{*}$ Data set 3 . The s.p.e. of the active $3 \hbar \omega$ shell were taken from the experimental energies of ${ }^{56} \mathrm{Ni}$, and the $2 \hbar \omega$ and $4 \hbar \omega$ shell energies were taken from the harmonic oscillator energies with $\hbar \omega / \mathrm{MeV}=45 A^{1 / 3}-25 A^{2 / 3}$. Fit1 was employed to adjust the experimental $\Delta^{N(Z)}$ for ${ }^{56} \mathrm{Fe}$.

Adjusting the particle-hole couplings $r$ and $p$

In the particle-hole matrix element $F$, defined in Eq. (3.5), the couplings $v_{s}$ and $v_{t}$ appear as linear combinations $v_{s}+v_{t}$ and 


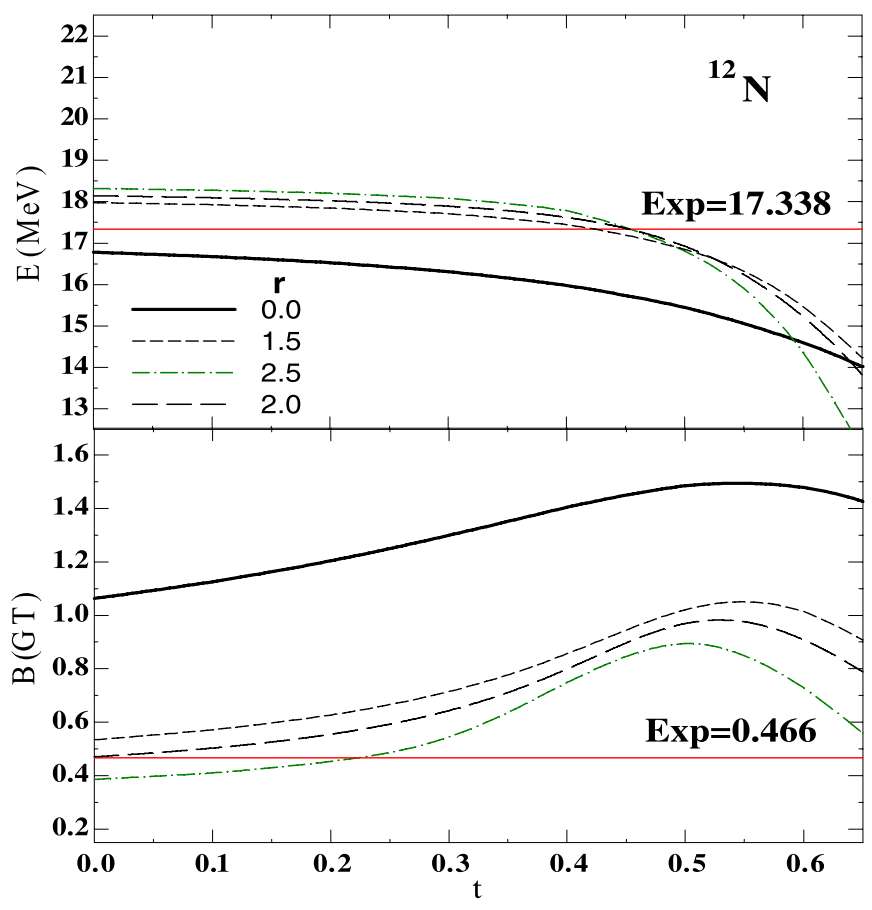

Fig. 1. Ground state energy E, and $B(G T)$-value in ${ }^{12} \mathrm{~N}$ for different couplings $r$ in the ph-channel, as a function of the $p p$-channel coupling $t$. The experimental data $[51,58,59]$ are also shown. The value $r=2$ corresponds to $v_{s}^{p h}=27$ a nd $v_{t}^{p h}=64$ (case PII of [23]). The Data set 2 was employed.

$3 v_{t}-v_{s}$. Therefore, it is convenient to introduce the dimensionless parameters

$r=\frac{v_{s}^{p h}+v_{t}^{p h}}{2 v_{s}^{\text {pair }}}, \quad p=\frac{3 v_{t}^{p h}-v_{s}^{p h}}{2 v_{s}^{\text {pair }}}$,

where

$v_{s}^{\text {pair }}=\frac{v_{s}^{\text {pair } N}+v_{s}^{\text {pair } P}}{2}$.

Moreover, to start we can use $v_{s}^{p h}=27$ and $v_{t}^{p h}=64$ (in units of $\mathrm{MeV} \mathrm{fm}^{3}$ ). These values are inferred from the systematic study of energetics of the GT resonances done by Nakayama et al. [56] (see also Ref. [44]), and have been extensively used in the QRPA calculations of the $\beta \beta$-decay in ${ }^{48} \mathrm{Ca}[25,30,57]$. Moreover, it makes sense to take the singlet ph coupling to be equal to $v_{s}^{\text {pair }}$, obtained from the proton and neutron gap equations, i.e.,

$v_{s}^{p h}=v_{s}^{\text {pair }}$.

\section{Adjusting the particle-particle couplings $s$ and $t$}

Here also it is convenient to normalize to $v_{s}^{\text {pair }}$ the coupling constants $v_{s}^{p p}$ and $v_{t}^{p p}$ that appear in the $p p$ matrix elements $G$ in Eq. (3.5), and correspond, respectively to the channels ( $T=1, S=$ $0)$ and $(S=1, T=0)$, i.e.,

$s=\frac{v_{s}^{p p}}{v_{s}^{\text {pair }}}, \quad t=\frac{v_{t}^{p p}}{v_{s}^{\text {pair }}}$.

For nuclei with $N>Z$ the pp-couplings are fixed on the basis of the SU(4) and isospin symmetry, as $v_{s}^{p p} \equiv v_{s}^{\text {pair }}$, and $v_{t}^{p p} \gtrsim v_{s}^{p p}[25$, 30]. However, in Ref. [22] it was shown that this parametrization might not be suitable for $N=Z$. In fact, the best agreement with data in ${ }^{12} \mathrm{C}$ was obtained when the $p p$-channel is totally switched off, i.e., $v_{s}^{p p} \equiv v_{t}^{p p}=0$, and three different set of values for the $p h$ coupling strengths were used. These conditions are related with

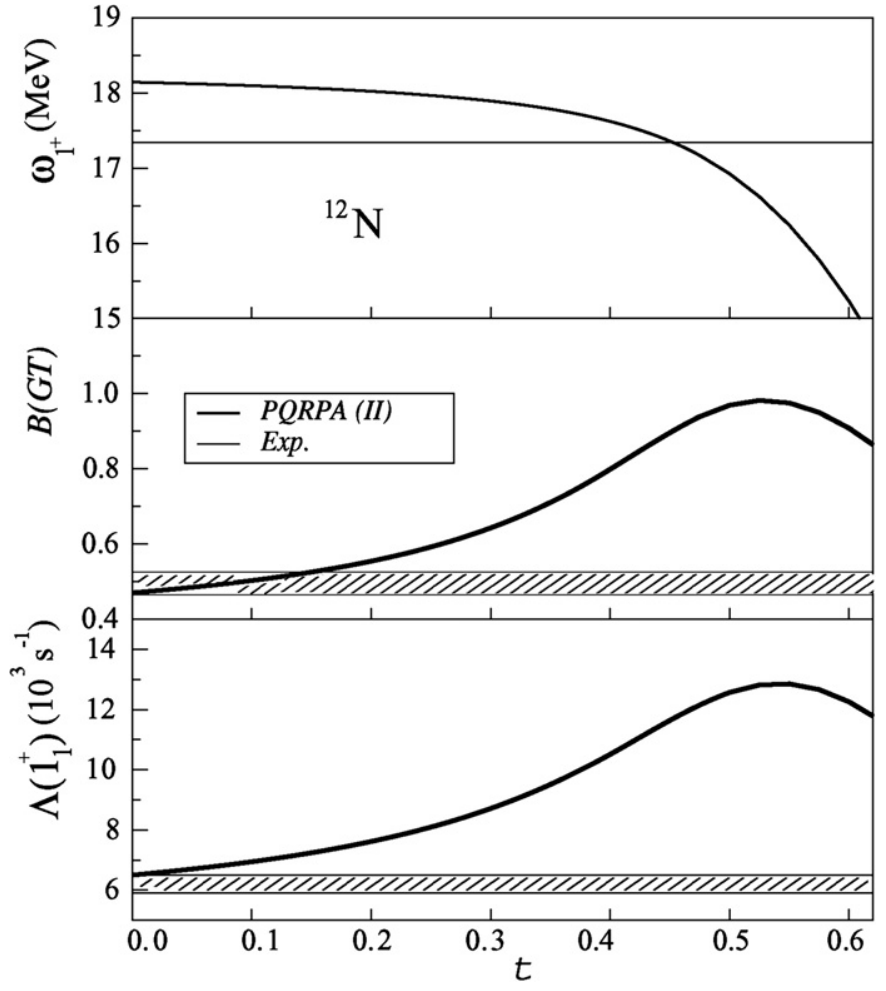

Fig. 2. Results from PQRPA calculations obtained in Ref. [23], as a function of the $p p$ parameter $t$, compared with the experimental data taken from Refs. [51,58,59], for: (i) ground state energy in ${ }^{12} \mathrm{~N}$ (upper panel), (ii) $B(G T)$-value for the $\beta$ transition in ${ }^{12} \mathrm{~N}$ (middle panel), and (iii) exclusive muon capture rate $\Lambda\left(1_{1}^{+}\right)$in ${ }^{12} \mathrm{~B}$ (lower panel). The parameters of the $p h$ channel for the $\delta$-interaction are $v_{s}^{p h}=27$ and $v_{t}^{p h}=64$.

$s=t$ for ${ }^{12} \mathrm{C}(N=Z)$, and with $s=1$ and $t$ variable in nuclei with $N>Z$. For ${ }^{56} \mathrm{Fe}$ were adopted the values $s=1$ and $t=0$. In the code QRAP the following conditions are standard: (i) $s=t$ with $t$ as a variable parameter for $N=Z$; and (ii) $s=1$, and $t$ as a variable parameter for $N>Z$, i.e., the residual interaction is defined as a function of two adjustable parameters $v_{t}^{p h}$ and $t$.

Several experimental data are available in the literature that can be used for fixing the residual interaction coupling constants, such as: ground state energies of daughter nuclei, $B(G T)$-values for the $\beta^{+}$or $\beta^{-}$decay, and partial muon capture rate $[51,58,59]$.

One can use the reduced space of six levels to identify in the output file, the quantities shown in Figs. 1 and 2. The results for three values of $t$ are shown in Table 3 .

The values of $\omega_{\mu}\left(1_{f}^{+}\right)$, and $S_{\mu}\left(1_{f}^{+}\right)$in ${ }^{12} \mathrm{~N}$ and ${ }^{12} \mathrm{~B}$ can be found in the output file AUXI.OUT. In the present case the largest value of index $f$ is $f_{\max }=16$. Both set of states, with $\mu=+1$, and $\mu=-1$, are ordered from highest to lowest energies. In the PQRPA, the most collective ones are that of the corresponding ground states: $\left|1_{F=16}^{+}\right\rangle$in ${ }^{12} \mathrm{~N}$ (and $\left|1_{F=17}^{+}\right\rangle$in ${ }^{12} \mathrm{~B}$ ) although there also are significant strengths in the states $F=7,11$, and 14. In QRPA, the ground state is in $\left|1_{F=16}^{+}\right\rangle$for both ${ }^{12} \mathrm{~N}$ and ${ }^{12} \mathrm{~B}$. These wave functions are presented below. For the PQRPA case, we also show the unperturbed energies $\omega_{\mu}^{0}\left(p n 1^{+}\right)$(which are not ordered), and the corresponding single-particle GT strengths $S_{\mu}^{0}\left(p n 1^{+}\right)$, given respectively, by (3.8), and (3.30) for the GT operator $\sigma$. The largest ones are $S_{+1}^{0}\left(1 p_{1 / 2}^{\pi}, 1 p_{3 / 2}^{v} ; 1^{+}\right)$, and $S_{-1}^{0}\left(1 p_{3 / 2}^{\pi}, 1 p_{1 / 2}^{v} ; 1^{+}\right)$, which in the particle-hole limit correspond to excitations $1 p_{3 / 2}^{v} \rightarrow 1 p_{1 / 2}^{\pi}$, and $1 p_{3 / 2}^{\pi} \rightarrow 1 p_{1 / 2}^{v}$. For spins and parities $J_{f}^{\pi} \neq 1^{+}$, or $0^{+}$, in AUXI.OUT are shown the energies $\omega_{\mu}$, but not the strengths $S_{\mu}$.

The results for the eigenvalue problem are displayed in the output file OUT.OUT. For the option $\mathrm{MAPR}=1$ are printed 
Table 3

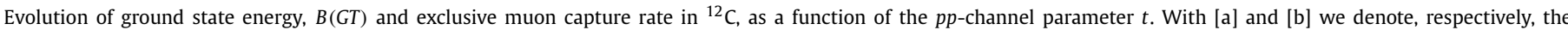

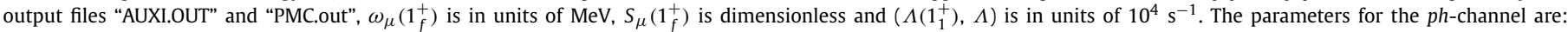
$v_{s}^{p h}=27, v_{t}^{p h}=64$.

\begin{tabular}{|c|c|c|c|c|c|c|}
\hline & \multirow[t]{2}{*}{ State [file] } & \multirow[t]{2}{*}{ Observable } & \multicolumn{4}{|l|}{$t$} \\
\hline & & & 0.0 & 0.3 & 0.6 & Exp. \\
\hline \multirow[t]{2}{*}{${ }^{12} \mathrm{~N}$} & 16 [a] & $\omega_{+1}\left(1_{16}^{+}\right)$ & 18.319 & 17.951 & 14.970 & $17.34[51]$ \\
\hline & 16 [a] & $S_{+1}\left(1_{16}^{+}\right)$ & 0.496 & 0.696 & 0.840 & $0.466[58]$ \\
\hline \multirow[t]{4}{*}{${ }^{12} \mathrm{~B}$} & 1 [a] & $\omega_{-1}\left(1_{16}^{+}\right)$ & 12.528 & 12.126 & 9.202 & $13.36[51]$ \\
\hline & 1 [a] & $S_{-1}\left(1_{16}^{+}\right)$ & 0.502 & 0.693 & 0.837 & $0.526[58]$ \\
\hline & 16 [b] & $\Lambda_{+1}\left(1_{16}^{+}\right)$ & 0.689 & 0.936 & 1.119 & $0.62(3)[59]$ \\
\hline & [b] & $\sum_{f} \Lambda_{+1}\left(1_{f}^{+}\right)$ & 1.722 & 1.537 & 1.183 & \\
\hline
\end{tabular}

out the matrix elements $(\mathcal{A}, \mathcal{B})$ (Eq. (3.25)) for the QRPA, or $\left(A_{\mu=1}, B\right)$ (Eq. (3.7)) for PQRPA. The nuclear wave functions $\left(X\left(p n ; J_{F}\right), Y\left(p n ; J_{F}\right)\right)$ are grouped to four, with the index $F$, defined in (3.14), going from 1 to $f_{\max }$ in the QRPA case, and from 1 to $2 f_{\max }$ in the PQRPA case. To make easy reading together with each set of wave functions are also printed: the value of $f$, the two quasiparticle configurations ( $p$ and $n$ ), and the unperturbed and perturbed energies.

Recalling Eqs. (3.8), (3.12), and (3.13) for the energies, one discovers without difficulty that within PQRPA:

1) The ground state in ${ }^{12} \mathrm{~N}$, with energy $\omega_{+1}\left(1^{+}\right)=18.319 \mathrm{MeV}$, has $f=F=16$, and that its wave function is:

$$
\begin{aligned}
\left.{ }^{12} \mathrm{~N}\right\rangle= & 0.963\left|1 p_{3 / 2}^{\pi} 1 p_{1 / 2}^{v}, 1^{+}\right\rangle+0.232\left|1 p_{3 / 2}^{\pi} 1 p_{3 / 2}^{\nu}, 1^{+}\right\rangle \\
& +0.122\left|1 p_{1 / 2}^{\pi} 1 p_{3 / 2}^{v}, 1^{+}\right\rangle+0.105\left|1 p_{1 / 2}^{\pi} 1 p_{1 / 2}^{v}, 1^{+}\right\rangle \\
& +\cdots .
\end{aligned}
$$

2) The ground state in ${ }^{12} \mathrm{~B}$, with energy $\omega_{-1}\left(1^{+}\right)=12.528 \mathrm{MeV}$, has $f=16, F=17$, and that its wave function is:

$$
\begin{aligned}
\left|{ }^{12} \mathrm{~B}\right\rangle= & -0.971\left|1 p_{1 / 2}^{\pi} 1 p_{3 / 2}^{\nu}, 1^{+}\right\rangle+0.204\left|1 p_{3 / 2}^{\pi} 1 p_{3 / 2}^{\nu}, 1^{+}\right\rangle \\
& -0.125\left|1 p_{3 / 2}^{\pi} 1 p_{1 / 2}^{v}, 1^{+}\right\rangle+0.090\left|1 p_{1 / 2}^{\pi} 1 p_{1 / 2}^{v}, 1^{+}\right\rangle \\
& +\cdots
\end{aligned}
$$

One proceeds in a similar way for the QRPA output, with energies now given by Eqs. (3.23) and (3.24). Now, the ground state energies in ${ }^{12} \mathrm{~B}$, and ${ }^{12} \mathrm{~N}$, are, respectively, $\omega_{-1}\left(1^{+}\right)=12.437 \mathrm{MeV}$, and $\omega_{+1}\left(1^{+}\right)=17.992 \mathrm{MeV}$, while the wave function for both nuclei is:

$$
\begin{aligned}
\left|1_{16}^{+}\right\rangle= & -0.272\left|1 p_{3 / 2}^{\pi} 1 p_{1 / 2}^{\nu}, 1^{+}\right\rangle-0.759\left|1 p_{3 / 2}^{\pi} 1 p_{3 / 2}^{\nu}, 1^{+}\right\rangle \\
& +0.356\left|1 p_{1 / 2}^{\pi} 1 p_{3 / 2}^{v}, 1^{+}\right\rangle-0.472\left|1 p_{1 / 2}^{\pi} 1 p_{1 / 2}^{v}, 1^{+}\right\rangle \\
& +\cdots
\end{aligned}
$$

From the comparison of the wave functions (4.9), and (4.11) it can be easily figure out why Volpe et al. [21] called attention to "difficulties in choosing the ground state of ${ }^{12} \mathrm{~N}$, because the lowest state is not the most collective one" when the QRPA is used. This is an important issue that clearly gives you an idea about the need for the number projection. In fact, as seen from (4.9), and (4.11), the PQRPA yields the correct one-particle-one-hole $(1 \mathrm{p} 1 \mathrm{~h})$ limits $1 p_{3 / 2}^{\pi} \rightarrow 1 p_{1 / 2}^{v}$ and $1 p_{3 / 2}^{v} \rightarrow 1 p_{1 / 2}^{\pi}$, for ${ }^{12} \mathrm{~N}$, and ${ }^{12} \mathrm{~B}$ ground states, respectively. All remaining configurations come from the higher order $2 \mathrm{p} 2 \mathrm{~h}$, and $3 \mathrm{p} 3 \mathrm{~h}$ excitations. Contrary, the QRPA state (4.11) is dominantly the two-hole excitation $\left[\left(1 p_{3 / 2}^{\pi}\right)^{-1},\left(1 p_{3 / 2}^{v}\right)^{-1}\right]$, which corresponds to the ground state of ${ }^{10} \mathrm{~B}$. This should not be a surprise, as we know that the proton-neutron QRPA states are the same for all four nuclei ${ }^{12} \mathrm{~N},{ }^{10} \mathrm{~B},{ }^{14} \mathrm{~N}$, and ${ }^{12} \mathrm{~B}$. More details on this question can be found in [23, Fig. 3]. The $1 \mathrm{p} 1 \mathrm{~h}$ amplitudes $\left[\left(1 p_{3 / 2}^{\pi}\right)^{-1}, 1 p_{1 / 2}^{v}\right]$, and $\left[\left(1 p_{3 / 2}^{v}\right)^{-1},\left(1 p_{1 / 2}^{\pi}\right)\right]$ are dominantly present in the QRPA states $f=13$, and $f=15$, i.e.,

$$
\begin{aligned}
\left|1_{13}^{+}\right\rangle= & -0.476\left|1 p_{3 / 2}^{\pi} 1 p_{1 / 2}^{v}, 1^{+}\right\rangle+0.437\left|1 p_{3 / 2}^{\pi} 1 p_{3 / 2}^{v}, 1^{+}\right\rangle \\
& +0.441\left|1 p_{1 / 2}^{\pi} 1 p_{3 / 2}^{v}, 1^{+}\right\rangle-0.096\left|1 p_{1 / 2}^{\pi} 1 p_{1 / 2}^{v}, 1^{+}\right\rangle \\
& +\cdots, \\
\left|1_{15}^{+}\right\rangle= & 0.703\left|1 p_{3 / 2}^{\pi} 1 p_{1 / 2}^{v}, 1^{+}\right\rangle+0.708\left|1 p_{1 / 2}^{\pi} 1 p_{3 / 2}^{v}, 1^{+}\right\rangle \\
& +\cdots
\end{aligned}
$$

The wave functions displayed above clearly evidence the superiority of the PQRPA on the QRPA.

\section{Output for the $v$-nucleus processes}

The output of the results for the weak processes is selected according to the value of IREAC:

IREAC $=\mathbf{0}$ prints the results for the muon capture rate in the file (QMC.out or PMC.out). For $J^{\pi}=0^{+}$or $J^{\pi}=1^{+}$are shown in this output file the folded strengths $\widetilde{S}_{\mu}\left(J_{f}, E\right)$ ( $\mathrm{S}^{\wedge}$ TILDE) defined by (3.32), where 'ENERGY' represents $E$. The partial capture rate for each state $f$, the perturbed energy $\omega_{\mu=-1}$, and the strength $S_{\mu=-1}\left(J_{f}\right)$ (if $J^{\pi}=0^{+}$, or $J^{\pi}=1^{+}$) are shown in the table labeled CAPTURE RATE. The total capture for the evaluated spin $J^{\pi}$ is presented in the last line.

IREAC $=\mathbf{1}$ or IREAC $=\mathbf{2}$ prints the results for the neutrino or antineutrino cross sections in the files (QNC.out/PNC.out) or (QAC.out/PAC.out). We repeat in this output the folded strengths $\widetilde{S}_{\mu}\left(J_{f}, E\right)$ (3.32) for $J^{\pi}=0^{+}$or $J^{\pi}=1^{+}$. The cross sections (SIGMA(Enu)) are calculated as a function of the neutrino energy (Enu) for each nuclear spin from $f=1$ to $f=f_{\max }$. The perturbed $\omega_{\mu= \pm 1}$ energies for the daughter nucleus are also shown according the process related. The absolute value of maximum $(\cos \theta=-1)$ and minimum $(\cos \theta=1)$ nuclear momentum transfer $(|k|)$ in units of $\mathrm{MeV} / \mathrm{c}$ for each energy are also printed.

Note: The cross sections are printed up to a maximum energy of $250 \mathrm{MeV}$. Depending upon the single particle space employed, the cross sections, as a function of the neutrino energy, should be restricted to lower energies. This issue will be discussed and explained in details in a next work [60]. Anyway, the PQRPA cross sections obtained within the single-particle space provided as examples are well behaved up to $E_{v / \bar{v}}<100 \mathrm{MeV}$ on averaged according to ${ }^{*}$ Data set $1,{ }^{*}$ Data set 2 and ${ }^{*}$ Data set 3 . This interval of energies is important for supernova neutrinos and low-energy decay-at-rest neutrinos [10].

\section{Routines included with the code}

QRPA solves the pn-QRPA or pn-PQRPA charge-exchange problem for a nuclear spin $J_{f}^{\pi}$ of the daughter odd-odd nucleus. 
SUAVE calculates and prints the folded strength $J^{\pi}=0^{+}$or $J^{\pi}=1^{+}$given by Eq. (3.32), folding the $\widetilde{S}_{\mu}\left(J_{f}\right)$ strength with a Lorentzian function with $\eta=1 \mathrm{MeV}$.

RMUONCAP calculates the muon capture rate given by formula (2.29).

SIMPSN2 calculates the neutrino or antineutrino cross sections as a function of the neutrino energy. This subroutine uses the function $\mathbf{G}$ to call the subroutine SECCION, which evaluates the cross section formula (2.23) using the Gauss-Legendre $N$-point quadrature formula [61] on the function $\mathbf{F}$ to evaluate the angular integration of the transition amplitude times $E_{\ell}$.

MATRIXP computes the matrix elements with the delta residual interaction given in Ref. [62] for the PQRPA. The matrix elements were modified according to the projection procedure shown in Eq. (3.7).

MATRIX computes the matrix elements with the delta residual interaction given in Ref. [62] for the QRPA. The matrix elements are shown in Eqs. (3.25).

RPA finds eigenvalues and eigenvectors for the QRPA or PQRPA equations. It uses the subroutine EIGRF and other related subroutines from the IMSL Library [63] to orthonormalize the eigenvectors.

GAPII solves the set of BCS coupled equations (3.17) and (3.18) to obtain the $v_{k}$ and $u_{k}$ for neutrons and protons.

CONFGT builds up the pn configurations for a given spin and parity.

FAUX evaluates the particle-particle matrix elements $G\left(k k k^{\prime} k^{\prime}\right.$; 0 ) and $F\left(k k k^{\prime} k^{\prime} ; 0\right)$, which are used to solve the gap equations for neutrons and protons using the delta interaction.

RADWF computes harmonic oscillator radial wave functions. It uses the additional subroutine OSCILL to evaluate the radial coefficients.

HYBRD finds a zero of a system of $N$ nonlinear equations in $N$ variables by a modification of the Powell Hybrid method. This subroutine was provided by the Argonne National Laboratory [55]. It uses the subroutine FCN to calculate the PBCS nonlinear equations given by formula (3.1).

FIPERMAT evaluates the perturbed matrix elements for the weak decay operator, according to Eq. (3.27) for PQRPA, and Eq. (3.29) for QRPA. The radial part of the SPNME were defined in Ref. [64]. This subroutine uses the subroutine ANGULARMATRIX to calculate the angular part of single-particle matrix elements defined in Ref. [57], and shown in Appendix A for the sake of completeness.

There are other routines in the code that are shortly described as follows. PRINMA prints the matrix elements $(\mathcal{A}, \mathcal{B})$ for the QRPA, or $\left(A_{\mu}, B\right)$ for PQRPA, SKIPCOM is used to skip comments in the input file, UNPMOM3 evaluates the unperturbed projected matrix elements for beta decay, BETMAT2 is used to calculate the single-particle matrix elements for beta decay, PROENER calculates the quantities for the projected quasiparticles energies in (3.10).

\section{Things to do}

1. Use the sample input Data set 1 to obtain the results presented in Table 3.

2. Modify the input Data set 1 by Data set 2 , setting all parameters of the residual interaction to zero. These values correspond to BCS or PBCS approximation. Compare the folded strength of Data set 1 with Data set 2 shown in Fig. 4 of Ref. [23].

3. In Ref. [65] the s.p.e. for neutrons were changed to analyze the systematics of the paring strength in the odd carbon isotopes. Change the s.p.e. for neutrons in Data set 2 and reproduce the systematics shown in the level scheme of Fig. 2 and the spectroscopic factors of Fig. 3 of Ref. [65].
4. Compare the QRPA and PQRPA results for the exclusive $v_{e}-{ }^{12} \mathrm{C}$ cross section, as a function of the neutrino energy, with the DAR experimental data from Ref. [68]. Note that the QRPA result is not collective, and the addition of other $1^{+}$cross sections (for example, that of states (4.12)) is required to get agreement with the experimental value.

\section{Acknowledgements}

This work was partially supported by the U.S. DOE grants DEFG02-08ER41533 and DE-FC02-07ER41457 (UNEDF, SciDAC-2), and the Research Corporation. F.K. thanks the CONICET-Argentina for the financial support through PIP-6159 and PIP-0377. A.R.S. wishes to express sincere thanks to C.A. Barbero and A.E. Mariano for help received in programming the sp matrix elements.

\section{Appendix A. Single-particle nuclear matrix elements}

The elementary operators defined in Eq. (2.16) have the reduced single-particle $p n$ matrix elements (RSPME) defined in [57,41] (recall that $\kappa=|\mathbf{k}|$ and $\mathbf{p}=-i \nabla)$.

For the RSPME dependent on the tensor product of spherical harmonic times the nucleon velocity we have

$$
\begin{aligned}
\left\langle p,\left(l_{p} \frac{1}{2}\right), j_{p}|| j_{\mathrm{L}}(\kappa r)\left[Y_{\mathrm{L}}(\hat{\mathbf{r}}) \otimes \nabla\right]_{\mathrm{J}}|| n,\left(l_{n} \frac{1}{2}\right), j_{n}\right\rangle \\
=\frac{(-1)^{1+\mathrm{J}+\mathrm{L}}}{\sqrt{4 \pi}}\left[W_{\mathrm{LJ}}^{(-)}(p n) R_{\mathrm{L}}^{(-)}(p n ; \kappa)\right. \\
\left.\quad+W_{\mathrm{LJ}}^{(+)}(p n) R_{\mathrm{L}}^{(+)}(p n ; \kappa)\right],
\end{aligned}
$$

with angular and radial parts, respectively:

$$
\begin{aligned}
& W_{\mathrm{LJ}}^{( \pm)}(p n)= \pm(-1)^{l_{p}+j_{n}+\mathrm{J}+1 / 2} \hat{\mathrm{J}} \hat{\mathrm{L}} \hat{l}_{p} \hat{j}_{p} \hat{j}_{n}\left(l_{n}+\frac{1}{2} \mp \frac{1}{2}\right)^{1 / 2} \\
& \times\left(l_{p} \mathrm{~L} \mid l_{n} \mp 1\right)\left\{\begin{array}{ccc}
l_{p} & j_{p} & \frac{1}{2} \\
j_{n} & l_{n} & \mathrm{~J}
\end{array}\right\}\left\{\begin{array}{ccc}
\mathrm{L} & \mathrm{J} & 1 \\
l_{n} & l_{n} & \mp 1 l_{p}
\end{array}\right\}, \\
& R_{\mathrm{L}}^{( \pm)}(p n ; \kappa)=\int_{0}^{\infty} u_{n_{p}, l_{p}}(r)\left(\frac{d}{d r} \pm \frac{2 l_{n}+1 \pm 1}{2 r}\right) \\
& \times u_{n_{n}, l_{n}}(r) j_{\mathrm{L}}(\kappa r) r^{2} d r .
\end{aligned}
$$

We use here the angular coupling $\left|\left(\frac{1}{2}, l\right) j\right\rangle, \hat{J} \equiv \sqrt{2 J+1}$ and $\left(l_{p} L \mid l_{n} \mp 1\right)$ is the short notation for the Clebsh-Gordon coefficient $\left(l_{p}\right.$ OLO $\left.\left(l_{n} \mp 1\right) 0\right)$.

For the scalar product of spin times nucleon velocity, we have

$$
\begin{aligned}
\langle p, & \left.\left(l_{p} \frac{1}{2}\right), j_{p}|| j_{\mathrm{J}}(\kappa r) Y_{\mathrm{J}}(\hat{\mathbf{r}})(\sigma \cdot \nabla)|| n,\left(l_{n} \frac{1}{2}\right), j_{n}\right\rangle \\
= & \frac{1}{\sqrt{4 \pi}}\left[W_{\mathrm{J}}^{(-)}(p n) R_{\mathrm{J}}^{(-)}(p n ; \kappa)\right. \\
& \left.+W_{\mathrm{J}}^{(+)}(p n) R_{\mathrm{J}}^{(+)}(p n ; \kappa)\right],
\end{aligned}
$$

with the angular part

$$
\begin{aligned}
W_{\mathrm{J}}^{( \pm)}(p n)= & \pm(-1)^{l_{n}+j_{n}+\mathrm{J}+1 / 2} \sqrt{6} \hat{\mathrm{J}} \hat{l}_{p} \hat{j}_{p} \hat{j}_{n}\left(l_{n}+\frac{1}{2} \mp \frac{1}{2}\right)^{1 / 2} \\
& \times\left(l_{p} \mathrm{~J} \mid l_{n} \mp 1\right)\left\{\begin{array}{ccc}
1 & \frac{1}{2} & \frac{1}{2} \\
j_{n} & l_{n} & l_{n} \mp 1
\end{array}\right\}\left\{\begin{array}{ccc}
l_{n} \mp 1 & j_{n} & \frac{1}{2} \\
j_{p} & l_{p} & \mathrm{~J}
\end{array}\right\},
\end{aligned}
$$

being the radial part $R_{\mathrm{J}}^{( \pm)}(p n ; \kappa)$ as in (A.2). 
The RSPME of the two operators independent of the nucleon velocity is written below. For the spherical harmonic operator we have

$$
\begin{aligned}
\langle p & \left.,\left(l_{p} \frac{1}{2}\right), j_{p}|| j_{\mathrm{J}}(\kappa r) Y_{\mathrm{J}}(\hat{\mathbf{r}})|| n,\left(l_{n} \frac{1}{2}\right), j_{n}\right\rangle \\
& =\frac{1}{\sqrt{4 \pi}} W_{\mathrm{J} 0}(p n) R_{\mathrm{J}}^{0}(p n ; \kappa),
\end{aligned}
$$

with the angular and radial parts, respectively:

$$
\begin{aligned}
& W_{\mathrm{J} 0}(p n)=(-1)^{j_{p}-j_{n}} \hat{\jmath}_{j} \hat{j}_{n}\left(\begin{array}{ccc}
j_{p} & j_{n} & \mathrm{~J} \\
\frac{1}{2} & -\frac{1}{2} & 0
\end{array}\right), \\
& R_{\mathrm{J}}^{0}(p n ; \kappa)=\int_{0}^{\infty} u_{n_{p}, l_{p}}(r) u_{n_{n}, l_{n}}(r) j_{\mathrm{J}}(\kappa r) r^{2} d r .
\end{aligned}
$$

Finally, the RSPME dependent on the tensor product of spherical harmonic times the spin operator reads

$$
\begin{aligned}
& \left\langle p,\left(l_{p} \frac{1}{2}\right), j_{p}|| j_{\mathrm{L}}(\kappa r)\left[Y_{\mathrm{L}}(\hat{\mathbf{r}}) \otimes \sigma\right] J|| n,\left(l_{n} \frac{1}{2}\right), j_{n}\right\rangle \\
& =\frac{(-1)^{\mathrm{L}+1+\mathrm{J}}}{\sqrt{4 \pi}} W_{\mathrm{LJ}}(p n) R_{\mathrm{L}}^{0}(p n ; \kappa),
\end{aligned}
$$

where the angular part is

$$
\begin{aligned}
W_{\mathrm{LJ}}(p n)= & (-1)^{l_{p}} \sqrt{6} \hat{j}_{p} \hat{j}_{n} \hat{l}_{p} \hat{l}_{n} \hat{j}_{p} \hat{\mathrm{L}} \hat{\mathrm{J}} \\
& \times\left(\begin{array}{ccc}
l_{p} & \mathrm{~L} & l_{n} \\
0 & 0 & 0
\end{array}\right)\left\{\begin{array}{ccc}
\frac{1}{2} & l_{p} & j_{p} \\
\frac{1}{2} & l_{n} & j_{n} \\
1 & \mathrm{~L} & \mathrm{~J}
\end{array}\right\},
\end{aligned}
$$

with the radial part $R_{\mathrm{L}}^{0}(p n ; \kappa)$ given by (A.6).

\section{Appendix B. Fermi function and effective momentum approximation (EMA)}

To account for the Coulomb interaction between the charged lepton and the residual nucleus, the QRAP code is setup to use by default the Fermi function $[45,46]$. This correction was employed in several works for reactions on ${ }^{12} \mathrm{C}$ with neutrinos from the DAR of $\mu^{+}$. As pointed out in Ref. [21], the quantity $p_{\ell} R_{A}$ is of the order of 0.5 , where $p_{\ell}$ is the lepton momentum, and $R_{A}$ is the radius of the nucleus. Thus, the correction is well described by a Fermi function. Yet, for high energy neutrinos, e.g. neutrinos from the DIF of $\pi^{+}$, the outgoing muons have $p_{\ell} R_{A}>0.5$. For these relativistic leptons, the effective momentum approximation (EMA) [66] should take care of the Coulomb field of the daughter nucleus, instead of the Fermi function. This prescription for the Coulomb correction is considered in the code within the subroutine SECCION. More precisely, with $E M A=0$ the Fermi function is employed, while with $\mathrm{EMA}=1$ the EMA prescription is used. In the EMA procedure, the lepton energy and momentum are modified by a constant electrostatic potential within the nucleus

$E_{\ell, e f f}=E_{\ell}-V_{\text {eff }}, \quad p_{\ell, e f f}=\sqrt{E_{\ell, e f f}^{2}-m_{\ell}^{2}}$,

with $V_{e f f}=4 V_{C}(0) / 5=-6 Z_{f} \alpha / 5 R_{A}[38,67]$. These two approximations for the Coulomb correction were tested in the calculation of the inclusive cross section for neutrino scattering on ${ }^{208} \mathrm{~Pb}$ [38]. As shown in Ref. [38], the Fermi function correction overestimates the cross sections at higher neutrino energies where the EMA provides a more reliable approach. Thus, we recommend to use the Fermi function correction in the range of neutrino energies for which the cross section is below the corresponding EMA value, whereas the EMA could be employed at higher energies, as shown in previous studies $[21,38]$.

As a final comment, the QRPA code could be easily extended to calculate $v_{\mu}$-induced processes. This was done in Refs. [22,23] to calculate $v_{\mu}-{ }^{12} \mathrm{C}$ cross sections using the EMA prescription for the DIF regime of the LSND experiment. The nuclear structure calculations remain the same, while the kinematics changes by changing the electron mass to the muon mass in the variable RMLEP.

\section{References}

[1] A. Aguilar, et al., LSND Collaboration, Phys. Rev. D 64 (2001) 112007.

[2] Y. Fukuda, et al., Super-Kamiokande Collaboration, Phys. Rev. Lett. 81 (1998) 1562;

Y. Ashie, et al., Super-Kamiokande Collaboration, Phys. Rev. Lett. 93 (2004) 101801.

[3] B. Aharmim, et al., SNO Collaboration, Phys. Rev. C 59 (2005) 055502; M.B. Smy, et al., Super-Kamiokande Collaboration, Phys. Rev. D 69 (2004) 011104

[4] T. Araki, et al., KamLAND Collaboration, Phys. Rev. Lett. 94 (2005) 081801.

[5] M.H. Ahn, et al., K2K Collaboration, Phys. Rev. Lett. 90 (2003) 041801.

[6] G. McLaughlin, G.M. Fuller, Astrophys. J. 455 (1995) 202.

[7] Y.-Z. Qian, J. Wassweburg, Phys. Rep. 442 (2007) 237.

[8] N.Yu. Agafonova, et al., Astroparticle Phys. 27 (2007) 254.

[9] R. Maschuw, et al., KARMEN Collaboration, Prog. Part. Nucl. Phys. 40 (1998) 183 , and references mentioned therein.

[10] B. Armbruster, et al., KARMEN Collaboration, Phys. Rev. D 65 (2002) 112001.

[11] R.C. Allen, et al., Phys. Rev. Lett. 64 (1990) 1871.

[12] D.A. Krakauer, et al., Phys. Rev. C 45 (1992) 2450.

[13] C. Athanassopoulos, et al., LSND Collaboration, Phys. Rev. C 54 (1996) 2685; C. Athanassopoulos, et al., LSND Collaboration, Phys. Rev. Lett. 77 (1996) 3082

[14] C. Athanassopulus, et al., LSND Collaboration, Phys. Rev. C 58 (1998) 2489; C. Athanassopulus, et al., LSND Collaboration, Phys. Rev. Lett. 81 (1998) 1774.

[15] Y. Efremenko, Nucl. Phys. B 138 (Proc. Suppl.) (2005) 343; F.T. Avignone III, Y.V. Efremenko, J. Phys. G 29 (2003) 2615.

[16] S.E. Woosley, D. Hartmann, R.D. Hoffman, W.C. Haxton, Astrophys. J. 356 (1990) 272.

[17] P. Adamson, et al., MINOS Collaboration, Phys. Rev. D 76 (2007) 072005

[18] A.R. Samana, C.A. Bertulani, F. Krmpotić, arXiv:0808.1317v1 [nucl-th].

[19] E. Kolbe, K. Langanke, S. Krewald, Phys. Rev. C 49 (1994) 1122.

[20] J.S. Winfield, N. Anantaraman, Sam M. Austin, L.H. Harwood, J. van der Plicht, H.-L. Wu, A.F. Zeller, Phys. Rev. C 33 (1986) 1333.

[21] C. Volpe, N. Auerbach, G. Colò, T. Suzuki, N. Van Giai, Phys. Rev. C 62 (2000) 015501.

[22] F. Krmpotić, A. Mariano, A. Samana, Phys. Lett. B 541 (2002) 298.

[23] F. Krmpotić, A. Mariano, A. Samana, Phys. Rev. C 71 (2005) 044319.

[24] A. Samana, F. Krmpotić, A. Mariano, R. Zukanovich Funchal, Phys. Lett. B 642 (2006) 100;

A. Samana, F. Krmpotić, A. Mariano, R. Zukanovich Funchal, Phys. Rev. C 78 (2008) 024312.

[25] J. Hirsch, F. Krmpotić, Phys. Rev. C 41 (1990) 792.

[26] J. Hirsch, F. Krmpotić, Phys. Rev. C 41 (1990) 792.

[27] J. Hirsch, F. Krmpotić, Phys. Lett. B 246 (1990) 5.

[28] F. Krmpotić, J. Hirsch, H. Dias, Nucl. Phys. A 542 (1992) 85.

[29] F. Krmpotić, A. Mariano, T.T.S. Kuo, K. Nakayama, Phys. Lett. B 319 (1993) 393 and references therein.

[30] F. Krmpotić, Shelly Sharma, Nucl. Phys. A 572 (1994) 329.

[31] J.A. Hableib, R.A. Sorensen, Nucl. Phys. A 98 (1967) 542.

[32] P. Vogel, M.R. Zirnbauer, Phys. Rev. Lett. 57 (1986) 731.

[33] D. Cha, Phys. Rev. C 27 (1987) 2269.

[34] M.S. Yousef, V. Rodin, A. Faessler, F. Simkovic, Phys. Rev. C 79 (2009) 014314.

[35] A.R. Samana, C.A. Bertulani, Phys. Rev. C 78 (2008) 024312.

[36] E. Kolbe, K. Langanke, G. Martínez-Pinedo, Phys. Rev. C 60 (1999) 052801(R).

[37] R. Lazauskas, C. Volpe, Nucl. Phys. A 792 (2007) 219.

[38] N. Paar, D. Vretenar, T. Marketin, P. Ring, Phys. Rev. C 77 (2008) 024608.

[39] J. Toivanen, E. Kolbe, K. Langanke, G. Martínez-Pinedo, P. Vogel, Nucl. Phys A 694 (2001) 395.

[40] K. Langanke, G. Martínez-Pinedo, P. von Neumann-Cosel, A. Richter, Phys. Rev. Lett. 93 (2004) 202501.

[41] T.W. Donnelly, W.C. Haxton, Atomic Data and Nuclear Data Tables 23 (1979) 103; T.W. Donnelly, R.D. Peccei, Phys. Rep. 50 (1979) 1.

[42] J.D. Walecka, Theoretical Nuclear and Subnuclear Physics, Imperial College Press and World Scientific Publishing Co. Pte. Ltd., 2004.

[43] T. Kuramoto, M. Fukugita, Y. Kohyama, K. Kubodera, Nucl. Phys. A 512 (1990) 711.

[44] H. Castillo, F. Krmpotić, Nucl. Phys. A 469 (1987) 637. 
[45] R.J. Blin-Stoyle, S.C.K. Nair, Adv. Phys. 15 (1966) 493.

[46] H. Behrens, W. Bühring, Electron Radial Wave Functions and Nuclear Beta Decay, Clarendon, Oxford, 1982.

[47] D.J. Rowe, Nuclear Collective Motion, Methuen, London, 1970.

[48] A.M. Lane, J. Martorrel, Ann. Phys. 129 (1980) 273.

[49] A. Bohr, B.R. Mottelson, Nuclear Structure, vol. II, Benjamin, New York, 1975.

[50] F. Krmpotić, K. Ebert, W. Wild, Nucl. Phys. A 342 (1980) 497;

F. Krmpotić, K. Nakayama, A. Pio Galeão, Nucl. Phys. A 399 (1983) 478;

E. Bauer, F. Krmpotić, K. Nakayama, Nucl. Phys. A 485 (1988) 46.

[51] F. Ajzenberg-Selove, Nucl. Phys. A 433 (1985) 1.

[52] T. Suzuki, et al., Phys. Rev. C 35 (1987) 2212.

[53] W.-T. Chou, et al., Phys. Rev. C 47 (1993) 163.

[54] A. Bohr, B.R. Mottelson, Nuclear Structure, vol. I, Benjamin, New York/ Amsterdam, 1969.

[55] Burton S. Garbow, Kenneth E. Hillstrom, Jorge J. More, Minpack Project, Argonne National Laboratory, March 1980.
[56] K. Nakayama, A. Pio Galeão, F. Krmpotić, Phys. Lett. B 114 (1982) 217.

[57] C. Barbero, F. Krmpotić, D. Tadić, Nucl. Phys. A 628 (1998) 170;

C. Barbero, F. Krmpotić, A. Mariano, D. Tadić, Nucl. Phys. A 650 (1999) 485.

[58] D.E. Alburger, A.M. Nathan, Phys. Rev. C 17 (1978) 280.

[59] G.H. Miller, et al., Phys. Lett. B 41 (1972) 50.

[60] A.R. Samana, C.A. Bertulani, F. Krmpotić, N. Paar, in preparation.

[61] Subroutine GAULEG, Copr. 1986-92 Numerical Recipes Software A.

[62] I. Supek, Teorijska Fizika i Struktura Materije, Skolska Knjiga, Zagreb, 1992.

[63] IMSL Library Reference Manual, 8th ed., IMSL Inc, Houston, Texas, 1980. Available at: http://ib.cnea.gov.ar/ fiscom/Libreria/imsl_old.

[64] K. Ikeda, Prog. Theor. Phys. 31 (1964) 434.

[65] A.R. Samana, T. Tarutina, F. Krmpotić, M.S. Hussein, T.T.S. Kuo, Nucl. Phys. A 791 (2007) 36.

[66] J. Engel, Phys. Rev. C 57 (1998) 2004.

[67] A. Aste, D. Trautmann, Eur. Phys. J. A 33 (2007) 11

[68] C. Athanassopoulus, et al., LSND Collaboration, Phys. Rev. C 55 (1997) 2078. 